\title{
Current and turbulence measurements at the FINO1 offshore wind energy site: analysis using 5-beam ADCPs
}

\author{
Mostafa Bakhoday-Paskyabi ${ }^{1,2} \cdot$ Ilker Fer $^{1}$ - Joachim Reuder ${ }^{1}$
}

Received: 4 November 2016 / Accepted: 9 October 2017 / Published online: 6 November 2017

(c) The Author(s) 2017. This article is an open access publication

\begin{abstract}
We report concurrent measurements of ocean currents and turbulence at two sites in the North Sea, one site at upwind of the FINO1 platform and the other 200$\mathrm{m}$ downwind of the Alpha Ventus wind farm. At each site, mean currents, Reynolds stresses, turbulence intensity and production of turbulent kinetic energy are obtained from two bottom-mounted 5-beam Nortek Signature1000s, high-frequency Doppler current profiler, at a water depth of approximately $30 \mathrm{~m}$. Measurements from the two sites are compared to statistically identify the effects of wind farm and waves on ocean current variability and the turbulent structure in the water column. Profiles of Reynolds stresses are found to be sensible to both environmental forcing and the wind farm wake-induced distortions in both boundary layers near the surface and the seabed. Production of turbulent kinetic energy and turbulence intensity exhibit approximately similar, but less pronounced, patterns in the presence of farm wake effects.
\end{abstract}

Keywords 5-beam ADCP . Wave bias · Turbulence . Reynolds stresses $\cdot$ Alpha Ventus · FINO1

This article is part of the Topical Collection on the 18th Joint Numerical Sea Modelling Group Conference, Oslo, Norway, 10-12 May 2016

Responsible Editor: Martin Verlaan

Mostafa Bakhoday-Paskyabi

Mostafa.Bakhoday@nersc.no

1 Geophysical Institute, University of Bergen and Bjerknes Centre for Climate Research, Bergen, Norway

2 Present address: Nansen Environmental and Remote Sensing Center, Bergen, Norway

\section{Introduction}

Detailed knowledge of ocean circulation and the mechanisms responsible for turbulent mixing is of great importance in coastal oceanography (e.g. hydrodynamics, sediment transport, energy transport, marine ecology, contaminants spread and water quality) and in successful developments of offshore renewable energy sites (e.g. fatigue and transient loading on marine constructions). However, the ability to accurately quantify the characteristics and statistics of turbulence depends directly on high-resolution field observations which are typically sparse. The inherent noise in technology used, patchy and intermittent nature of turbulence, and hydrodynamic complexity of the surface boundary layer make it difficult to conduct successful and reliable measurements.

Velocity measurements are one of the matured techniques for statistical description of the natural turbulence. A single-point acoustic Doppler velocimeter (ADV) measures the three-dimensional velocity field at a small sampling volume with a high sampling frequency, $\geq 20 \mathrm{~Hz}$. The ability of an ADV to measure turbulence statistics depends on the distance of ADV sampling volume to the boundary (e.g. regions of strong turbulent intensity), the level of noise variances, contaminations induced by wave motions and non-stationarity of flow conditions. The single-point measurements from ADVs are obviously unable to capture spatial structures in the water column, and an array of ADVs is then required to provide high-resolution vertical profiles of ocean currents and small-scale velocity fluctuations. Furthermore, this intrusive measuring technique is highly sensitive to biofouling in shelf seas as well as flow distortion due to the sensor's head and its installation. In recent years, the development of free falling/rising microstructure profilers has facilitated the quality measurements of 
turbulence (e.g. dissipation rates of TKE, $\varepsilon$ ) throughout the water column. As a result of turbulence intermittency, however, the method requires repeated profiling, which is labour intensive and is limited to short-term measurement periods (Fer and Bakhoday-Paskyabi 2014). Observation capability has been expanded by the use of high-frequency acoustic Doppler current profilers (ADCPs) which collect extended profiles of turbulent quantities from the measured along-beam velocities. Compared to ADVs, ADCPs, however, cannot appropriately resolve short temporal (spatial) scales of motions because of Doppler noise (horizontal spreading of ADCP beams) and non-homogenous nature of flow. Lohrmann et al. (1990) used a pulse-to-pulse coherent ADCP on the continental shelf to measure Reynolds stresses and eddy viscosity by assuming that turbulence statistics are homogenous. Gargett (1994) used an ADCP to directly measure vertical velocity of water column and investigated the (horizontal) homogeneity assumption used in (indirect) turbulence measurements from the opposing beams of ADCP. She illustrated that the direct and indirect vertical velocity measurements begin to be less correlated by increasing distance from the instrument. Wiles et al. (2006) compared profiles of $\varepsilon$ from velocity microstructure with those measured from ADCP velocity profiles by applying a structure function technique which relies on stationary assumption of long-time averages. Osalusi et al. (2009) utilised ADCP measurements to determine several turbulence parameters such as TKE, rate of TKE production, Reynolds shear stresses and eddy viscosity using a so-called variance method (VM) for various tidal regimes. They further estimated $\varepsilon$ by applying structure function and inertial dissipation method to ADCP profiles (Thomson 2012; Bakhoday-Paskyabi et al. 2015). In summary, the aforementioned techniques have been well-suited for estimation of turbulence, but they are not fully applicable to regions with strong intermittent turbulence consisting of scales less than the system time-averaging interval and particularly in the presence of energetic surface gravity waves.

ADCPs, as a common ocean current profiling technique, use short-term bursts of along-beam velocities to extract vertical profiles of currents and turbulence parameters (Lu and Lueck 1999; Howarth and Souza 2005; Nystrom et al. 2007). In the presence of surface gravity waves, however, contributions from the wave orbital velocities may be an order of magnitude larger than the turbulence fluctuating velocities (Trowbridge 1998; Shaw and Trowbridge 2001; Gerbi et al. 2008). In such cases, even a small orientation of the instrument's head produces a large wave-induced bias in the estimated variances and covariances from ADCP measurements (Rosman et al. 2008). Therefore, several methods have been suggested and applied to accurately calculate Reynolds stresses using ADCPs in the presence of surface gravity waves (Rosman et al. 2008; Whipple et al. 2006; Kirincich et al. 2010).

The primary objective of the present study is to report current and turbulence measurements under varying surface forcing and flow conditions in the close vicinity of the FINO1 platform and Alpha Ventus wind farm in the North Sea. We use novel platforms including different oceanographic sensors, particularly Nortek 5-beam Signature1000s (hereafter S1000s) to link the upper ocean turbulence and current characteristics with disturbances induced by the offshore structures. We further aim to investigate the capability of the S1000 systems mounted on fixed bottom frames to derive the typical turbulence metrics in shallow seas in the presence of gravity wave orbital motions.

The paper is organised as follows: details of experimental sites and measuring strategies are given in Section 2. Measuring fundamentals of 5-beam S1000s to measure three-dimensional mean currents and turbulence metrics are presented in Section 3. Comparisons between measurements at two sites in the upwind direction of the FINO1 and downwind direction of the Alpha Ventus wind farm are carried out in Section 4. Some conclusions are given in the final section.

\section{Observations}

An experiment was carried out between June and November 2015 with assistance from the FINO and the Alpha Ventus wind farm operators, to investigate the physical processes of air-sea interaction, particularly turbulence generated by the breaking and non-breaking surface gravity waves, and coherent large-scale Langmuir circulations. The deployment was part of the Offshore Boundary Layer Experiment at the FINO1 (OBLEX-F1).

\subsection{Measurement site and instrumentations}

Ocean stratification, currents and turbulence were measured with four moorings deployed on 4 June 2015 (yearday 154 (YD154), where YD1 is the first day of January 2015) in the immediate vicinity of the research offshore platform FINO1 in the North Sea $\left(54^{\circ} 00^{\prime} 53.5^{\prime \prime} \mathrm{N}, 6^{\circ} 35^{\prime} 15.5^{\prime \prime} \mathrm{E}\right.$, hereafter S1) and the Alpha Ventus, the first German offshore wind farm, which is located to the east of FINO1 with a closest distance of 400-m (Fig. 1a). Two systems including a "Bottom Lander" (BL1, Fig. 2a) and a mooring were deployed in the southwest sector of FINO1 to preserve the less disturbed observations under the (quasi) free flow conditions, Fig. 1b. Another similar Bottom Lander (BL2) and an oceanographic mooring (OM) with horizontal separation of $100-\mathrm{m}$ were deployed in the northeast sector of the Alpha Ventus's 


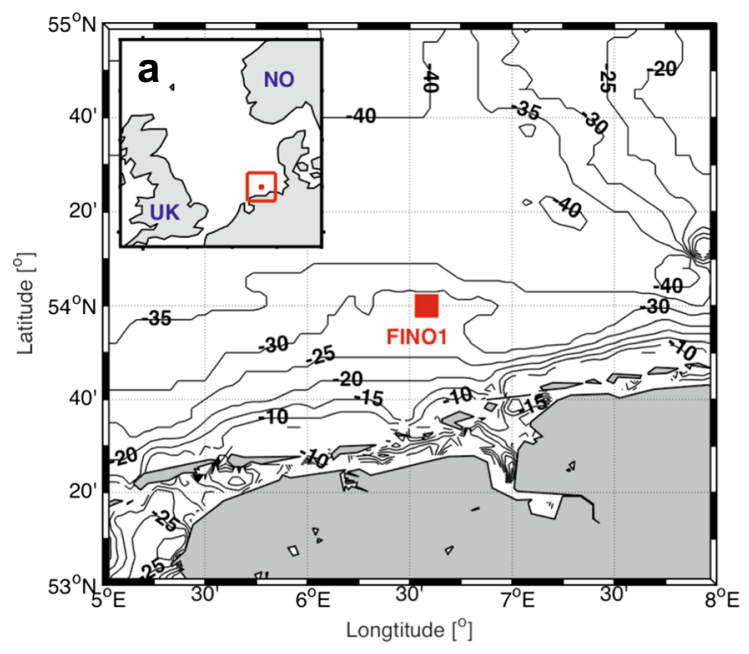

Fig. 1 a Regional map of deployment site at FINO1 in the North Sea. Contours show isobaths between 10 and $50 \mathrm{~m}$ depths. The inset map indicates the location of the OBLEX-F1 study site. b Layout of the

safety zone (hereafter S2) to make the same measurements as those performed at the location of S1, see Table 1 for details (Fig. 2). All moorings were recovered at 1 November 2015 (YD304) together with their bottom anchors. Each Bottom Lander at S1 and S2 is a tripod-shaped frame equipped with an upward pointing Nortek Vector current meter and an upward looking 5-beam S1000 with its first vertical bin at 2.8-m height above the seabed (Fig. 2c). The 5-beam S1000s carried out concurrent measurements of the velocity profile in averaged mode (as 2-min averages every 10-min at 1-m cells) and in turbulence mode (as 15-min bursts every hour at $0.5-\mathrm{m}$ cells with a sampling frequency of $4 \mathrm{~Hz}$ ). The cell size in turbulence mode is sufficient to resolve the important eddy scales.

The mooring deployed upwind of FINO1 is a bottomanchored Moored Autonomous Turbulence System (MATS) to measure the viscous dissipation rate, $\varepsilon$, at a fixed depth (Fer and Bakhoday-Paskyabi 2014) using a modified MicroRider turbulence package and an upward looking three-component Nortek Vector (Fig. 2c). The MATS's 22$\mathrm{m}$ tall mooring line was equipped with SeaBird (SBE56) temperature loggers at 5.5-, 9.5-, 13.5-, 15.5-, 17.5- and 21$\mathrm{m}$ heights above the seabed sampled at 3-s interval, two SeaBirds (SBE37) unpumped conductivity-temperaturedepth (CTD) loggers at 7.5- and 19.5-m heights above the seabed sampled at 1-min interval and a Seaguard current metre at 5.5-m height above the seabed sampled at 5-min interval.

In the Alpha Ventus safety zone, a similar bottom lander was deployed at a water depth of $31-\mathrm{m}$ with approximately 100-m distance from the OM system (Fig. 2b). The OM system measured the background currents, temperature and salinity downwind of the Alpha Ventus using a Seaguard

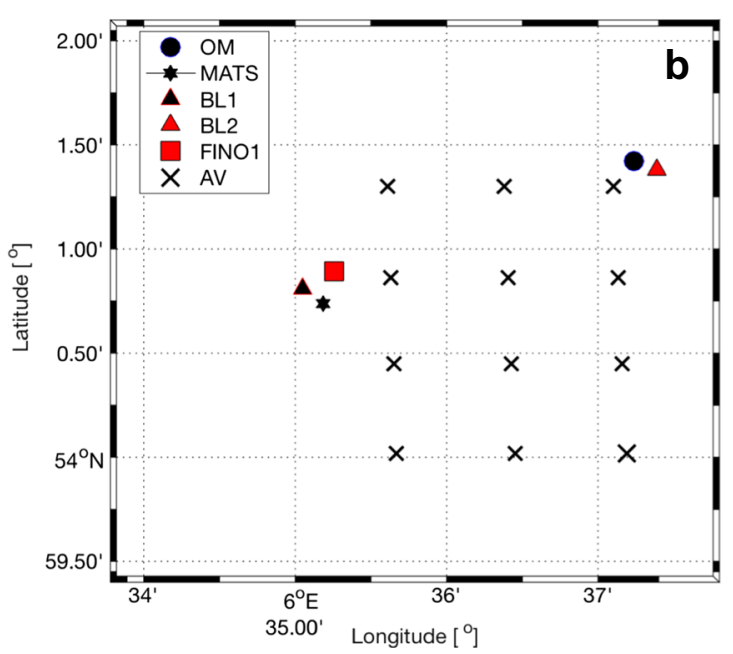

offshore wind farm Alpha Ventus (AV) and deployment locations relative to the FINO1 platform. Crosses indicate positions of AV's turbines

current metre (hereafter RCM) located at $5.5-\mathrm{m}$ height above the seabed sampled at 5-min interval, SBE56 temperature loggers at 5.5-, 8-, 9.6- and 11.2-m heights above the seabed sampled at 3-s interval and a Microcat CTD logger (unpumped) at 12.8-m height from the seabed sampled the background salinity and temperature at 1-min interval. Further details of instrumentations are provided in Table 2.

During deployment, the S1000 on BL1 experienced a transducer failure and stopped operating after 10 days with almost 3 days of records. Furthermore, all instruments were subject to intensive buildup of diverse organic sea life (biofouling) such as soft-bodied organisms and hard-bodied barnacle shells. The biofouling also constrained the quality data, in particular those collected from ADVs.

To examine the upper ocean variability in the area of offshore structures and to check the reliability of 5-beam measurements, we selected approximately 3 days of measurements during June 2015 when both S1000s on BL1 and BL2 returned high-quality data (Table 1). Unfortunately, ADVs are not included for the analysis considered in this study because of a series of sampling and technical issues. Therefore, the main focus of this study would be on measurements from the BL-mounted S1000s.

\subsection{Surface forcing measurements}

The 100-m meteorological mast of FINO1 has been instrumented with meteorological sensors to perform multi-level measurements of wind speed, wind direction, air temperature, humidity and air pressure since 2004. Here, we use data from a cup anemometer at 33-m height above the mean see 
a
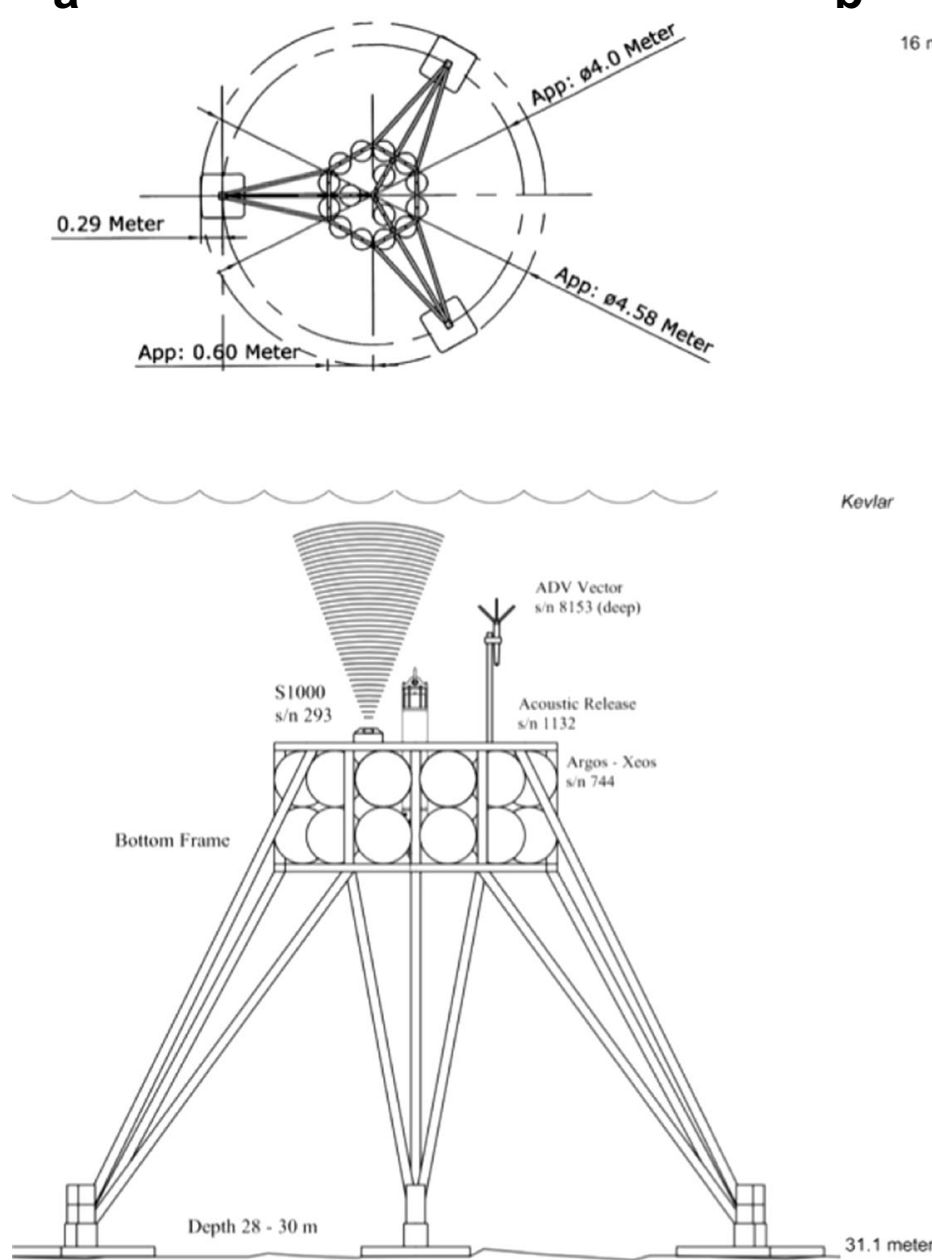

b

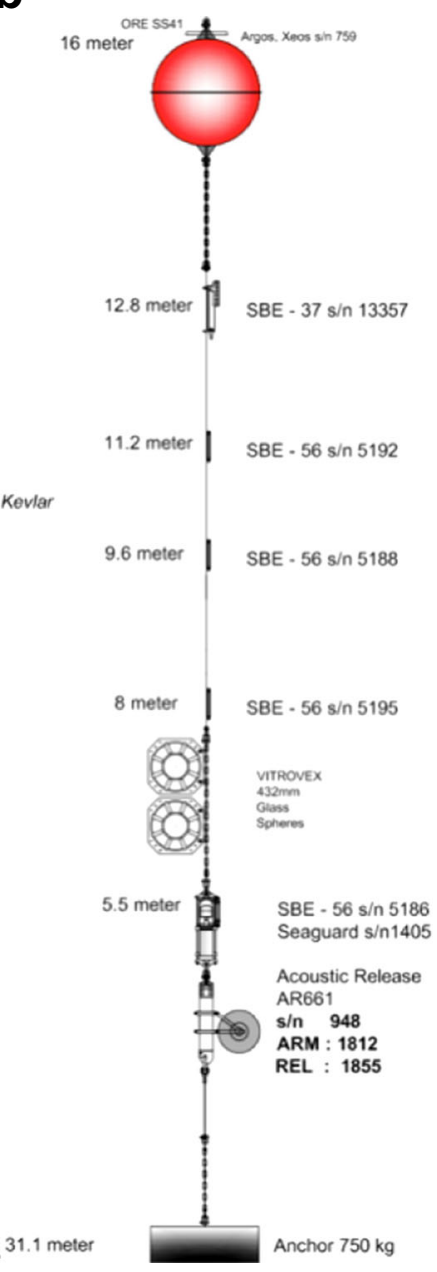

C

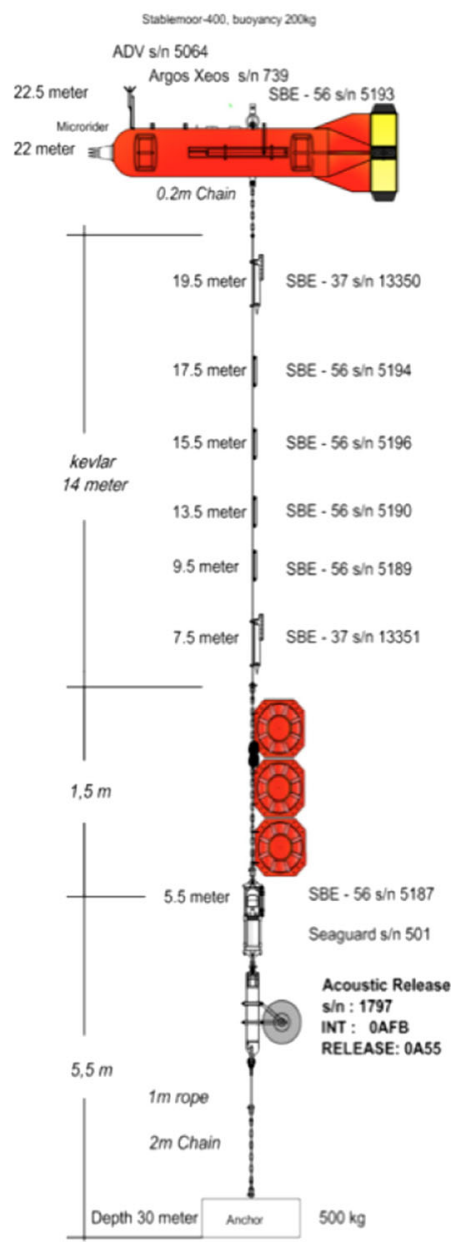

Fig. 2 a Bottom lander view from top (top) and sketch of the BL deployed on the seafloor equipped with an ADV and a S1000 (below). The BL includes a double axis gimbal allowing the S1000 for an approximately $20^{\circ}$ tilt in any direction. b A conventional Oceanographic

level in order to have appropriate estimate for the wind at 10-m height using the Monin-Obukhov similarity applicable for the neutral atmospheric boundary layer (Large and Pond 1981). Statistics of the sea-state (i.e. significant wave height, peak period and wave direction) have been extracted from a Waverider buoy moored few hundred metres northwest of the platform.
Mooring $(\mathrm{OM})$ to measure temperature, salinity and water velocity in the downwind direction of AV. c Schematic of the MATS deployed at the upwind direction of FINO1

\section{Data analysis and methods}

\subsection{Velocity description}

The current velocity data, collected in averaged- and burstmode from S1000s sampled at a finite number of vertical segments (cells or bins) throughout the water column are

Table 1 Deployments of moorings during the OBLEX-F1 campaign

\begin{tabular}{llllll}
\hline Mooring & Longitude & Latitude & Depth & Deployment time, UTC & Recovery time, UTC \\
\hline BL1 & $54^{\circ} 0.81^{\prime} \mathrm{N}$ & $6^{\circ} 35.051^{\prime} \mathrm{E}$ & 30 & $04.06 .201506: 59$ & $01.11 .201510: 07$ \\
MATS & $54^{\circ} 0.737^{\prime} \mathrm{N}$ & $6^{\circ} 35.183^{\prime} \mathrm{E}$ & 30 & $04.06 .201508: 36$ & $01.11 .201510: 20$ \\
BL2 & $54^{\circ} 1.378^{\prime} \mathrm{N}$ & $6^{\circ} 37.389^{\prime} \mathrm{E}$ & 31 & $04.06 .201510: 36$ & $01.11 .201513: 12$ \\
OM & $54^{\circ} 1.423^{\prime} \mathrm{N}$ & $6^{\circ} 37.237^{\prime} \mathrm{E}$ & 31 & $04.06 .201511: 40$ & $01.11 .201517: 11$ \\
\hline
\end{tabular}


Table 2 Operation details for S1000s, Here, $f_{s}$ and $\Delta z$ denote the sampling frequency and the bin resolution, respectively

\begin{tabular}{lllllll}
\hline Mooring & Instrument & SN & $f_{s}[\mathrm{~Hz}]$ & hab [m] & $\Delta z$ & Sampling duration [days] \\
\hline BL1 & S1000 & 151 & 4 & 2.66 & 0.5 & 3 \\
MATS & RCM & 501 & 0.0033 & 5.5 & n/a & 151 \\
OM & RCM & 1405 & 0.0033 & 5.5 & n/a & 151 \\
BL2 & S1000 & 293 & 4 & 2.66 & 0.5 & 151 \\
\hline
\end{tabular}

Note that the bin resolution for S1000s in average mode is 1-m. Height of each sensor above the seabed is presented by "hab". Furthermore, the sampling durations correspond to available raw datasets before applying the quality control criteria

quality controled to remove outliers, to account for the missing data and to accurately detect the air-water interface. The instantaneous current velocities in beam coordinate frame (for the turbulence-mode configuration) are then transformed into the Cartesian (Earth) system $u=(u, v, w)$, where $u$ (East), $v$ (North) and $w$ (Up) are horizontal and vertical velocities, respectively. For the sake of simplicity, we exclude effects of heading angle for all theoretical derivations in this section and account for its effect by using the heading rotation matrix afterward in order to perfectly align the instrument coordinate with the Earth coordinate system.

\subsection{Mean current measurements from $\mathrm{S} 1000$}

A five-beam S1000 consists of two pairs of opposing transducers (1-3 and 4-2 axes) and one transducer aligned with vertical axis of S1000 such that the beam 1 faces in the positive $x$ axis and beam 4 faces in the positive $y$-axis (Fig. 3a, c). The instrument divides each beam into a finite number of sampling volumes and calculates radial velocities at each sampling bin using the Doppler shift of acoustic sound waves. This results in estimates of five along-beam velocities $\left(\hat{b}_{1}, \hat{b}_{2}, \hat{b}_{3}, \hat{b}_{4}\right.$ and $\left.\hat{b}_{5}\right)$ defined to be positive towards the instrument. To be consistent with conventions used for most commercial ADCPs (Fig. 3b), we apply the following beam-mapping strategy, known as Janus configuration, Dewey and Stringer (2007):

$b_{1}=\hat{b}_{1} ; b_{2}=\hat{b}_{3} ; b_{3}=\hat{b}_{4} ; b_{4}=\hat{b}_{2} ;$ and $b_{5}=\hat{b}_{5}$,

and

$\phi_{1}=\hat{\phi}_{1} ; \phi_{2}=\hat{\phi}_{3} ;$ and $\phi_{3}=-\hat{\phi}_{2}$,

where $\hat{\phi}_{1}, \hat{\phi}_{2}$ and $\hat{\phi}_{3}$ are heading, pitch and roll angles of S1000, respectively (Fig. 3b, c). The heading of Janus
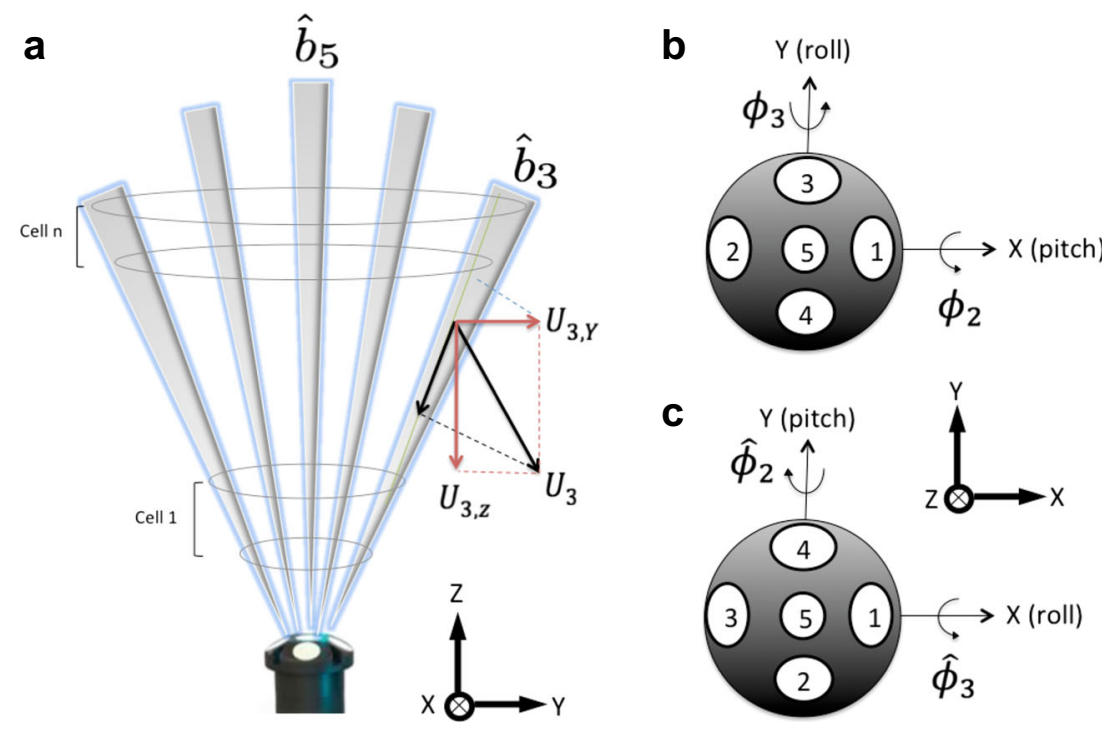

Fig. 3 a Side view of the five-beam S1000. The instrument XYZ coordinate system is drawn together with decomposition of the third alongbeam velocity, $U_{3}$, in the corresponding $\mathrm{Y}$ and $\mathrm{Z}$ components using the transducers' angles relative to the vertical axis. b Two pairs of opposing beams (1-2 and 3-4) reside in two perpendicular planes, $x-z$ and $y-z$, respectively. The beam-convention has been adapted from Dewey

and Stringer (2007); and $\mathbf{c}$ two pairs of opposing beams (1-3 and 4-2) for the $\mathrm{S} 1000$. Each beam makes an angle of $\beta=25^{\circ}$ with the vertical axis for S1000's configuration. The heading, pitch and roll angles of the $\mathrm{S} 1000$ are $\hat{\phi}_{1}, \hat{\phi}_{2}$ and $\hat{\phi}_{3}$, respectively. Correspondingly, $\phi_{1}, \phi_{2}$ and $\phi_{3}$ are the heading, pitch and roll angles of the Janus ADCP as used in Dewey and Stringer (2007) 
ADCP is $\phi_{1}$ and its pitch and roll angles are $\phi_{2}$ and $\phi_{3}$, respectively. When S1000 experiences non-zero pitch, $\hat{\phi}_{2}$, and roll, $\hat{\phi}_{3}$, motions, the estimated along-beam tilted velocity profiles in the Janus orthogonal coordinate frame, XYZ, using Eqs. 1 and 2 are trigonometrically calculated from

$$
\begin{aligned}
b_{1} & =-U_{1, X} \sin \left(\beta+\phi_{3}\right)+U_{1, Y} \cos \left(\beta+\phi_{3}\right) \sin \left(\phi_{2}\right)-U_{1, Z} \cos \left(\beta+\phi_{3}\right) \cos \left(\phi_{2}\right), \\
b_{2} & =U_{2, X} \sin \left(\beta-\phi_{3}\right)+U_{2, Y} \cos \left(\beta-\phi_{3}\right) \sin \left(\phi_{2}\right)-U_{2, Z} \cos \left(\beta-\phi_{3}\right) \cos \left(\phi_{2}\right), \\
b_{3} & =-U_{3, X} \cos (\beta) \sin \left(\phi_{3}\right)-U_{3, Y}\left[\sin (\beta) \cos \left(\phi_{2}\right)-\cos \left(\phi_{3}\right) \sin \left(\phi_{2}\right) \cos (\beta)\right] \\
& -U_{3, Z}\left[\cos (\beta) \cos \left(\phi_{2}\right) \cos \left(\phi_{3}\right)+\sin (\beta) \sin \left(\phi_{2}\right)\right], \\
b_{4} & =-U_{4, X} \sin \left(\phi_{3}\right) \cos (\beta)+U_{4, Y}\left[\sin (\beta) \cos \left(\phi_{2}\right)+\cos (\beta) \cos \left(\phi_{3}\right) \sin \left(\phi_{2}\right)\right] \\
& -U_{4, Z}\left[\cos \left(\phi_{2}\right) \cos \left(\phi_{3}\right) \cos (\beta)-\sin \left(\phi_{2}\right) \sin (\beta)\right], \\
b_{5} & =-U_{5, X} \sin \left(\phi_{3}\right)+U_{5, Y} \sin \left(\phi_{2}\right) \cos \left(\phi_{3}\right)-U_{5, Z} \cos \left(\phi_{2}\right) \cos \left(\phi_{3}\right),
\end{aligned}
$$

where $\beta$ denotes the slant angle of $\mathrm{S} 1000$ relative to the vertical $\left(25^{\circ}\right.$ for $\left.\mathrm{S} 1000\right)$ and $U_{i, X}, U_{i, Y}$ and $U_{i, Z}$ are respectively $X, Y$, and $Z$ velocity components at beam $i$ for $i=$ 1, 2, ․ 5 (Fig. 3a). Equations 3 and 7 contain 5 known radial velocities and 15 unknown velocity components. The above underdetermined system of equations can be solved by assuming horizontally homogeneous velocity field (i.e. $U_{X}=U_{i, X}, U_{Y}=U_{i, Y}$ and $\left.U_{Z}=U_{i, Z}\right)$. In matrix notation, Eqs. 3-7 under the horizontal homogeneity assumption and in the absence of (instrumental/environmental) noise effects can be written as

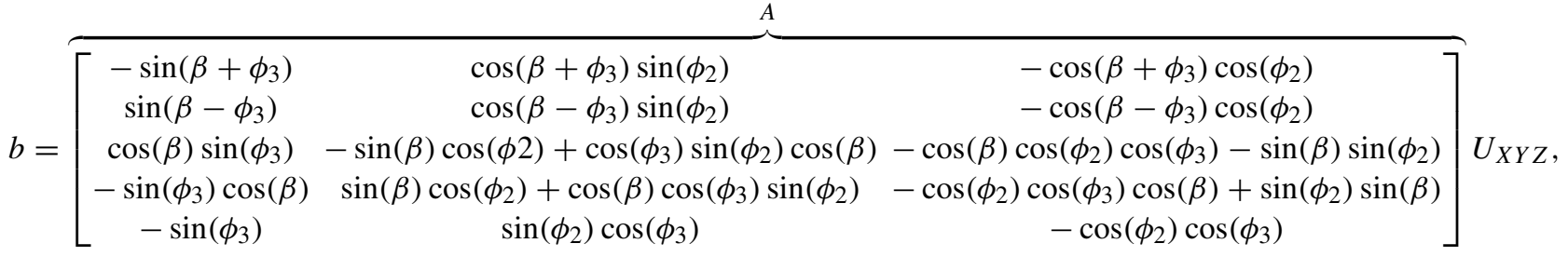

where $U_{X Y Z}=\left[U_{X}, U_{Y}, U_{Z}\right]$ denotes the velocity in the $X Y Z$ coordinate. The velocity vector in Earth frame (East/North/Up), $U_{E N U}=(u, v, w)$, is then determined as

$b=A U_{X Y Z}=A M U_{E N U}$,

where $T=A M$ is the transformation matrix and $M\left(\phi_{1}, \phi_{2}, \phi_{3}\right)$ is the total coordinate orientation matrix as a function of instrument heading, pitch and roll angles (for a rotation from the reference coordinate to the instrument frame). The total rotation matrix is calculated by multiplying the individual rotations about $z, x$ and $y$ axes, respectively (Dewey and Stringer 2007). Note that the order of three successive rotations is important in calculation of $M$. However, the error due to the order of rotation would be negligible for conditions where roll and pitch angles are small. Furthermore, we solve the overdetermined system (9) for $U_{X Y Z}$ as

$U_{X Y Z}=\left(A^{T} A\right)^{-1} A^{T} b$.
By solving systems (8) and (9) using Eq. 10, three components of velocity either in XYZ frame or ENU coordinate system are obtained. In this study, we use zero-tilt estimate for the matrix $A$ based on the value of $\beta$. This matrix is directly provided by the $\mathrm{S} 1000$ software as a standard postprocessing output for (zero-tilt) four-beam configuration (Lohrmann et al. 1990).

\subsection{Reynolds stresses using the variance method}

In this section, we assume that the velocity field is statistically homogeneous (spatial uniformity) in order to calculate the second-order turbulent quantities such as Reynolds stresses (RSs). For the rest of this paper, we use the ADCP configuration as shown in Fig. $3 \mathrm{~b} ; u, v$ and $w$ are assumed to be in the Earth coordinates, and we consider the local turbulent velocity as $u^{\prime}=u-\bar{u}$ (the same for other components), where overbar denotes some temporal averaging over scales much longer than the temporal scales of turbulent fluctuations. $\ddot{u}^{\prime}, \ddot{v}^{\prime}$, and $\ddot{w}^{\prime}$ are the velocity fluctuations in the instrument coordinate system. 
For an upward looking 5-beam S1000 with zero-tilt, the beam variances for the first four radial velocity fluctuations (i.e. $b_{i}^{\prime}=b_{i}-\bar{b}_{i}$ for $i=1, \cdots, 4$ in 4-beam configuration) in the instrument frame are written as

$\overline{b_{1}^{\prime 2}}=\overline{\ddot{u}^{\prime 2}} \sin ^{2} \beta+\overline{\ddot{w}^{\prime 2}} \cos ^{2} \beta+2 \overline{\ddot{u}^{\prime} \ddot{w}^{\prime}} \sin \beta \cos \beta$,

$\overline{b_{2}^{\prime 2}}=\overline{\ddot{u}^{\prime 2}} \sin ^{2} \beta+\overline{\ddot{w}^{\prime 2}} \cos ^{2} \beta-2 \overline{\ddot{u}^{\prime} \ddot{w}^{\prime}} \sin \beta \cos \beta$,

$\overline{b_{3}^{\prime 2}}=\overline{\ddot{v}^{\prime 2}} \sin ^{2} \beta+\overline{\ddot{w}^{\prime 2}} \cos ^{2} \beta+2 \overline{\ddot{v}^{\prime} \ddot{w}^{\prime}} \sin \beta \cos \beta$,

$\overline{b_{4}^{\prime 2}}=\overline{\ddot{v}^{\prime 2}} \sin ^{2} \beta+\overline{\ddot{w}^{\prime 2}} \cos ^{2} \beta-2 \overline{\ddot{v}^{\prime} \ddot{w}^{\prime}} \sin \beta \cos \beta$.

Reynolds stresses (RSs) are then extracted from the difference between the variances of opposing beam velocities, i.e. variance method (VM), as

$$
\begin{aligned}
-\overline{\ddot{u}^{\prime} \ddot{w}^{\prime}} & =\frac{1}{2 \sin 2 \beta}\left(\overline{b_{2}^{\prime 2}}-\overline{b_{1}^{\prime 2}}\right), \\
-\overline{\ddot{v}^{\prime} \ddot{w}^{\prime}} & =\frac{1}{2 \sin 2 \beta}\left(\overline{b_{4}^{\prime 2}}-\overline{b_{3}^{\prime 2}}\right),
\end{aligned}
$$

where primes denote the velocity fluctuations around the mean due to only turbulent motions. In deriving above equations, the turbulence is assumed to be horizontally homogeneous over the beam-separation distance so that statistics of turbulence are the same for all diverged beams (i.e. first-order homogeneity). We further assume that beam 1 and beam 2 are in $x-z$ plane (i.e. Janus configuration in Fig. 3b). As the deployed S1000s are subject to tilts and motions (i.e. non-zero pitch and roll angles), the vertical beam velocity $b_{5}$ is not perfectly representative for the true vertical velocity, and it is necessary to account for the tilt-induced errors in RSs. Based on geometrical considerations and neglecting the effects of along-beam wave orbital velocities, the velocity variances (normal stresses) and covariances (shear stresses) with an appropriate time-averaging period (here 15-min) similar to Eqs. 11-14 are calculated using beam variances for non-zero pitch and roll angles in the level coordinate frame (i.e. the Earth coordinate system by applying pitch and roll rotation matrices) as Dewey and Stringer (2007):

$$
\begin{aligned}
& \overline{u^{\prime 2}}=\frac{-1}{4 \sin ^{6} \beta \cos ^{2} \beta}\left[-2 \sin ^{4} \beta \cos ^{2} \beta\left(\overline{b_{2}^{\prime 2}}+\overline{b^{\prime 2}}-2 \cos ^{2} \beta \overline{b^{\prime 2}}{ }_{5}\right)\right. \\
& \left.+2 \sin ^{5} \beta \cos \beta \phi_{3}\left(\overline{b_{2}^{\prime 2}}-\overline{b^{\prime 2}}\right)\right] \text {, } \\
& \overline{v^{\prime 2}}=\frac{-1}{4 \sin ^{6} \beta \cos ^{2} \beta}\left[-2 \sin ^{4} \beta \cos ^{2} \beta\left(\overline{b_{4}^{\prime 2}}+\overline{b_{3}^{\prime 2}}-2 \cos ^{2} \beta \overline{b^{\prime 2}}{ }_{5}\right)-2 \sin ^{4} \beta \cos ^{2} \beta \phi_{3}\left(\overline{b^{\prime 2}}{ }_{2}-\overline{b^{\prime 2}}{ }_{1}\right)\right. \\
& \left.+2 \sin ^{3} \beta \cos ^{3} \beta \phi_{3}\left(\overline{b_{2}^{\prime 2}}-\overline{b^{\prime 2}}\right)-2 \sin ^{5} \beta \cos \beta \phi_{2}\left(\overline{b^{\prime 2}}-\overline{b^{\prime 2}}{ }_{3}\right)\right] \text {, } \\
& \overline{w^{\prime 2}}=\frac{-1}{4 \sin ^{6} \beta \cos ^{2} \beta}\left[-2 \sin ^{5} \beta \cos \beta \phi_{3}\left(\overline{b_{2}^{\prime 2}}-\overline{b^{\prime 2}}\right)+2 \sin ^{5} \beta \cos \beta \phi_{2}\left(\overline{b^{\prime 2}}-\overline{b^{\prime 2}}{ }_{3}\right)\right. \\
& \left.-4 \sin ^{6} \beta \cos ^{2} \beta \overline{b_{5}^{\prime 2}}\right] \text {, } \\
& \overline{u^{\prime} w^{\prime}}=\frac{-1}{4 \sin ^{6} \beta \cos ^{2} \beta}\left[\sin ^{5} \beta \cos \beta\left(\overline{b_{2}^{\prime 2}}-\overline{b_{1}^{\prime 2}}\right)+2 \sin ^{4} \beta \cos ^{2} \beta \phi_{2}\left(\overline{b_{2}^{\prime 2}}+\overline{b_{1}^{\prime 2}}\right)\right. \\
& \left.-4 \sin ^{4} \beta \cos ^{2} \beta \phi_{3} \overline{b^{\prime 2}}-4 \sin ^{6} \beta \cos ^{2} \beta \phi_{2} \overline{u^{\prime} v^{\prime}}\right] \text {, } \\
& \overline{v^{\prime} w^{\prime}}=\frac{-1}{4 \sin ^{6} \beta \cos ^{2} \beta}\left[\sin ^{5} \beta \cos \beta\left(\overline{b^{\prime 2}}-\overline{b^{\prime 2}}\right)-2 \sin ^{4} \beta \cos ^{2} \beta \phi_{2}\left(\overline{b^{\prime 2}}+\overline{b^{\prime 2}}\right)\right. \\
& \left.+4 \sin ^{4} \beta \cos ^{2} \beta \phi_{3} \overline{b^{\prime 2}}+4 \sin ^{4} \beta \cos ^{2} \beta \phi_{3} \overline{u^{\prime} v^{\prime}}\right] \text {. }
\end{aligned}
$$

Here, it is assumed that second-order statistics of turbulence are uniform across the area between opposing beams. In Eqs. 20 and 21, there is still one unknown, $\overline{u^{\prime} v^{\prime}}$, that cannot be derived from the variance method. Dewey and Stringer (2007) have shown that this term is small and may be ignored for the initial estimate of RSs. Alternatively, we estimate this unknown term from the turbulent fluctuating components of the S1000 velocities in the Earth coordinates, i.e. Eq. 9. Using Eqs. 17-21, the TKE and anisotropy ratio are determined as

$$
\begin{aligned}
& \mathrm{TKE}=\frac{1}{4 \sin ^{2} \beta}\left[\left(\overline{b_{1}^{\prime 2}}+\overline{b_{2}^{\prime 2}}+\overline{b_{3}^{\prime 2}}+\overline{b_{4}^{\prime 2}}\right)-2\left(2 \cos ^{2} \beta-\sin ^{2} \beta\right) \overline{b_{5}^{\prime 2}}\right. \\
& \left.-(\cot \beta-1) \phi_{3}\left(\overline{b_{2}^{\prime 2}}-\overline{b_{1}^{\prime 2}}\right)\right] \text {, } \\
& \mathrm{ATKE}=\frac{2 \sin ^{2} \beta \overline{{b^{\prime 2}}_{5}^{2}}+\cot \beta \phi_{3}\left(\overline{b_{2}^{\prime 2}}-\overline{b_{1}^{\prime 2}}\right)-\cot \beta \phi_{2}\left(\overline{b_{4}^{\prime 2}}-\overline{b_{3}^{\prime 2}}\right)}{\overline{b_{1}^{\prime 2}}+\overline{b_{2}^{\prime 2}}+\overline{b_{3}^{\prime 2}}+\overline{b_{4}^{\prime 2}}-4 \cos ^{2} \beta \overline{{b^{\prime}}_{5}^{2}}+\Lambda},
\end{aligned}
$$


where

$\Lambda=\cot \beta \phi_{2}\left(\overline{b^{\prime 2}}-\overline{b^{\prime 2}}\right)+(1-2 \csc 2 \beta) \phi_{3}\left(\overline{b_{2}^{\prime 2}}-\overline{b_{1}^{\prime 2}}\right)$.

The shear production as a rate at which the energy is transferred from mean flow to turbulence is calculated from the estimated momentum fluxes as

$P_{s}=-\rho_{w}\left[\overline{u^{\prime} w^{\prime}} \frac{\partial \bar{u}}{\partial z}+\overline{v^{\prime} w^{\prime}} \frac{\partial \bar{v}}{\partial z}+\overline{w^{\prime} w^{\prime}} \frac{\partial \bar{w}}{\partial z}\right]$,

where $\rho_{w}$ is the seawater density estimated from the measured temperature and salinity and vertical gradients of horizontal/vertical velocities and RSs are calculated from four and five along-beam radial velocities, respectively. The averaging time interval used in the calculation of RS is 15min bursts that ensures averaging over large eddies in the energy-containing subrange. Furthermore, performance of the $\mathrm{VM}$ is deteriorated in the presence of surface gravity waves which can lead to large along-beam variances. Therefore, even in calm sea-state conditions, the wave-induced bias must be reduced.

\subsection{Wave bias correction}

Turbulence field in the ocean surface boundary layer is permanently affected by the kinematic and dynamic constraints imposed by the ocean surface gravity waves so that the instantaneous velocity observations (in the Earth coordinate) may be conceptually decomposed into mean $\bar{u}=(\bar{u}, \bar{v}, \bar{w})$, turbulence, $u^{\prime}=\left(u^{\prime}, v^{\prime}, w^{\prime}\right)$ and wave, $\tilde{u}=(\tilde{u}, \tilde{v}, \tilde{w})$, components:

$u=\bar{u}+u^{\prime}+\tilde{u}$,

where overbar and tilde denote time averages over period of each burst and wave, respectively. On the small scale, the wave-generated orbital velocities distort the background strain field through straining of the vorticiy lines of turbulence motions (Teixeira and Belcher 2002). On the large scale, the wave-generated mass transport along the direction of wave propagation (i.e., Stokes drift) interact with flow vorticity resulting in formation of Langmuir turbulence (Leibovich 1983). Hence, underneath the wavy air-sea interface, all turbulence velocity spectra exhibit a significant energy elevation about the dominant wave peak frequency, $f=f_{p}$, accompanied by a variance (covariance) enhancement at frequencies far away from $f_{p}$ (i.e. advection of turbulent eddies by wave orbital motion) for conditions when the ratio between the rms wave orbital velocity, $\tilde{\sigma}_{w}$, and the mean current speed, $u_{c}$, (i.e. $R=\tilde{\sigma}_{w} / u_{c}$ ) increases for values less than 2 . For $R>2$, in particular in the coastal ocean, the wave-related variances (covariances) are, however, appeared at frequencies below the wave band and the stress-carrying turbulent eddies may be appeared at frequencies that overlap with the wave band. These complexities in interactions of turbulence and waves significantly influence the quality of extraction of turbulence statistics, e.g. RSs, from the turbulence (co)spectra.

Using two spatially (horizontally/vertically) separated ADVs, Trowbridge (1998) presented a method to reduce the wave bias in RS estimates by assuming that the wave velocities are correlated over the flow field between two sensors, and a well-separated spectral gap exists between waves and turbulence. While the method has been successfully applied to estimate RSs in surface-zone and the outer-shelf bottom boundary layer, it exhibited poor performance in the presence of complex wave climates. Feddersen and Williams (2007) adapted the technique to reduce the wave-induced bias even further by utilising the ogive curve to check the validity of RS estimates. The techniques of Trowbridge (1998) and Shaw and Trowbridge (2001) have been recently modified by Rosman et al. (2008) and Whipple et al. (2006) for observations from ADCPs. They estimated the vertical attenuation of wave-induced velocities using linear wave theory before applying the variance method. The methods, however, become more biased when wave periods decrease and wave heights increase, in particular very close to the sea surface.

Substituting (25) into (17)-(21) and assuming uncorrelated turbulent motions lead to emergence of three motioninduced biases due to the instrument tilt, wave-induced stresses and interactions between instrument tilt and wave orbital velocities. In order to account for these errors, we reformulate the variance method in spectral domain based on one-sided velocity spectra, by the fast Fourier transformation (FFT). In this approach, the covariances (shear stresses) are computed by spectral-integration for frequencies well below the wave scales. For example, an estimate of the vertical RS (from 1-2 axis in Janus configuration) is given by

$\overline{u^{\prime} w^{\prime}}(z) \approx \int_{0}^{f_{c}^{l}(z)} \operatorname{Co}_{u w}(f) d f$

where $\mathrm{Co}_{u w}(f)$ is the observed cospectrum at frequency $f$ and depth $z$, and $f_{c}^{l}(z)$ characterises the lower frequency bound for the dominant wave energy scales at depth $z$. During the period of experiment, the corresponding cutoff frequency ranged from 0.05 (near the surface) to $0.1 \mathrm{~Hz}$ (near the bottom). Considering this depth-dependent variation of $f_{c}^{l}$, we determine the value of $f_{c}^{l}$ at each vertical level as $50 \%$ of the peak frequency of the corresponding vertical velocity spectrum for frequencies between 0.05 and $0.1 \mathrm{~Hz}$, in order to appropriately separate between the wave band and the below-wave frequencies (see Eq. 30). While the (surface/bottom) boundary layer thicknesses limit the size 
of large turbulent eddies, the choice of $f_{c}^{l}$ also constrains the scales of energy-containing motions. For instance, for values of $f_{c}^{l} \leq 0.1 \mathrm{~Hz}$, turbulent eddies with sizes $\geq 5 \mathrm{~m}$ are advected past the ADCP sampling volumes by a steady current of $u_{c}=0.5 \mathrm{~m} \mathrm{~s}^{-1}$. Using Eq. 26 results, however, in a theoretical underestimation of RS estimates for conditions where the energy-containing eddies are highly energetic and intermittent in the absence of stratification, and when there exist complex kinematic and dynamic interactions between wave orbital motions and local turbulence. According to the latter, the energy-containing eddies may overlap in frequency with scales associated with the wave motions, and contributions from the wave-induced stresses (the non-zero cross-correlation between the vertical and the horizontal wave orbital velocities, e.g. $\overline{\tilde{u} \tilde{w}}$ ) dominate significantly stress estimates (i.e. wave bias). In order to check the kinematic effects of wave advection on the estimated RSs using Eq. 26, we use analytical shapes for the turbulence (co)spectra in the framework of the kinematic model proposed by Lumley and Terray (1983) and extended by Rosman and Gerbi (2017).

In homogeneous and isotropic turbulence, the onedimensional turbulence frequency spectrum (cospectrum) in the presence of wave advection for $f \ll f_{p}$, if $\tilde{\sigma}_{w} k_{o} / f_{p}>$ $2 \pi$, is given by

$\operatorname{Co}_{u_{i} u_{j}}(f)=\exp \left(-\mu_{i j}\left(\frac{\tilde{\sigma}_{w}}{u_{c}}\right)^{2}\left(\frac{f}{f_{p}}\right)^{2}\right) \operatorname{Co}_{u_{i} u_{j}}^{\text {lf }}$,

where $i, j=1,2,3,\left(u_{1}, u_{2}, u_{3}\right)=(u, v, w), \mathrm{Co}_{u_{i} u_{j}}^{\text {lf }}$ are the spectral densities at the plateau of the (auto/co)spectrum for frequencies $f \ll f_{p}, k_{o}$ denotes the wavenumber related to the spatial scale of the energy-containing turbulent eddies, and $\mu_{i j}=\left(2 \pi \tilde{\sigma}_{w} / f_{p}\right)^{-2} \int \operatorname{Co}_{\tilde{u}_{i} \tilde{u}_{j}}(f) / f_{p}^{2} d f$ are coefficients depending on the shape of wave spectrum (here, part of the observed spectral densities resides within the wave-affected frequency band). The RSs estimated using Eq. 27 at each depth $z$ are then compared with those calculated from Eq. 26 to characterise the effects of non-steady wave advection on turbulence statistics at frequencies well below waves.

\subsection{Turbulence intensity}

Turbulence intensity (TI) is generally used as a metric to characterise the strength of turbulence with an extensive application in marine energy society as it is connected to amount of loading experienced by offshore platforms and is a required input for many commercial codes, such as FLUENT, to model tidal (wind) turbine performance (Thomson et al. 2012). TI is defined as the ratio between turbulent fluctuations and the mean flow. For example, the turbulence intensity for the $u$-component, $I_{u}$, is defined by

$I_{u}=\frac{\sigma_{u}}{\bar{u}}=\frac{\sqrt{\overline{\overline{u^{\prime 2}}}-\xi_{u}^{2}}}{\bar{u}}$,

where $\sigma_{u}$ is the standard deviation of $u^{\prime}$, and $\xi_{u}$ denotes an empirical noise related to the measurement uncertainty and is used, in the statistical sense, to account for the effects of Doppler noise. In pulse-coherent Doppler systems, $\xi_{u}$ depends on the sound speed magnitude in water, the observed velocity variance, the pulse separation time of sonar system and the magnitude of the correlation between two sequential received pings. It can be also approximated from the velocity variance at the tail of velocity spectrum. In this study, we use the variances for all velocity components from frequencies below $f_{c}^{l}$ using Eq. 26 because of significant interactions between wave orbital velocities and turbulence. Furthermore, turbulence intensities for other components of velocity are obtained similarly.

\section{Results}

All measured velocities from S1000s in the Earth coordinate frame are rotated into the along-shore, $v$, and cross-shore, $u$, orthogonal reference frame using principle axes determined from a long-term (hourly averaged) measurements from an uplooking $(600 \mathrm{kHz})$ ADCP with 15 vertical cells deployed

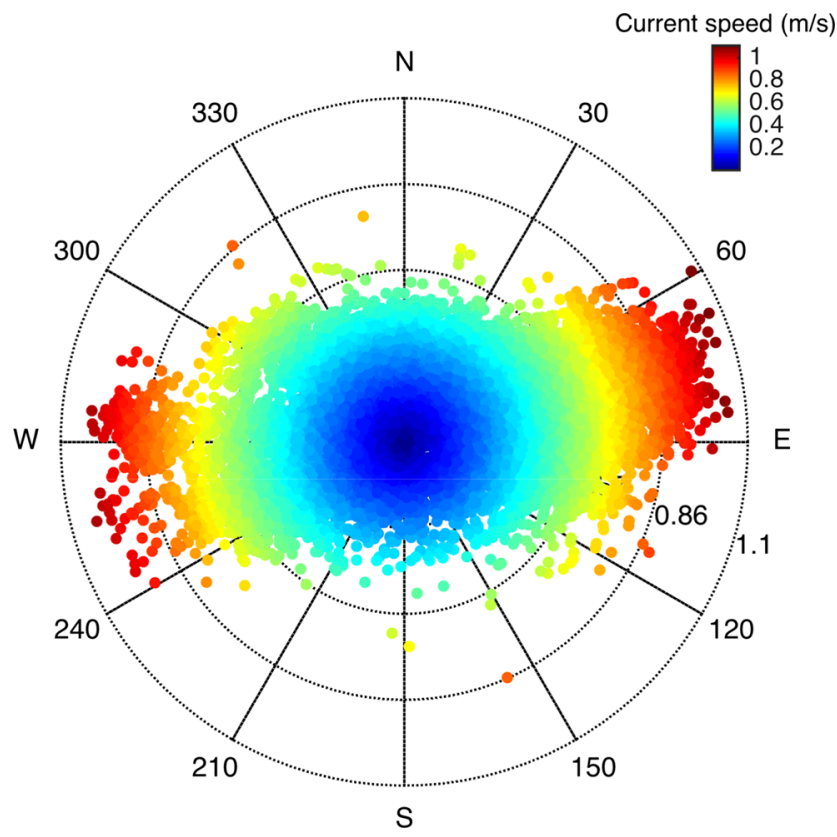

Fig. 4 The polar plot of the horizontal depth-averaged (over upper $8 \mathrm{~m}$ below the sea surface) velocity vector measured from an uplooking ADCP with 15 vertical bins deployed in the vicinity of FINO1, at upwind direction $\mathrm{x}$ 
near the FINO1 area, Fig. 4. An array of temperature sensors installed at the centre of the FINO1 jacket provided the ocean temperature at 3-, 10-, 15- and 20-m water depths, and the Sea Surface Temperature (SST) is determined from the temperature data at $3 \mathrm{~m}$ below the sea surface. These measurements combined with the temperature and salinity records from the MATS and the OM systems are used to compute the vertical density stratification (Fig. 6a, b). Comparisons between BL-mounted S1000s are performed between YD162 and YD165 when the BL1-mounted S1000 successfully logged data (Table 2).

\subsection{Environmental conditions}

Figure 5a illustrates wind speed and direction measurements at 33-m height scaled to $10-\mathrm{m}$ height based on MoninObukhov similarity. The wind speed rapidly increases from 6 to $11 \mathrm{~m} \mathrm{~s}^{-1}$ between June 12 and June 14 along with an abrupt change of wind direction from northeast sector to northwest sector. Air temperature is warmer than sea temperatures (stable atmospheric conditions) during June 2015 and the SST increases to $14.2{ }^{\circ} \mathrm{C}$ until at 3 rd of June where the wind speed increases to $>10 \mathrm{~m} \mathrm{~s}^{-1}$ (not shown). The significant wave height, $H_{s}$, varies between 0.4 and $1.9 \mathrm{~m}$, and the wave peak period varies between 4 and $9 \mathrm{~s}$ (Fig. 5b) for a period between June 12-14, 2015. At early YD162, the sea-state is dominated by short swell waves with a peak at $9 \mathrm{~s}$, in particular after YD163 together with a substantial decline of the peak wave period to $<5 \mathrm{~s}$. Figure $5 \mathrm{c}$ shows temporal evolution of wave age, $A_{w}=c_{p} / u_{* a}$, estimated from waves characterised at the peak of the wave energy spectrum. Here, $u_{* a}$ is the air-side friction velocity, and $c_{p}$ is the characteristic surface wave phase speed calculated from the dispersion relation for linear waves (Bakhoday-Paskyabi and Fer 2014). For small values of $A_{w}$, the wave growth rate is high and the wave field is strongly forced by the surface wind. For large values of $A_{w}$, the growth rates of waves are very small. In the vast majority of times, the sea-state is categorised as developed seas forced by the surface wind (i.e. $\left.A_{w}<80\right)$.

Figure 6 summarises the temperature, density and velocity structure during the experiment to give the context for interpretation of turbulence data from S1000s. The detailed temperature vertical structure between YD162YD180 shows typically well-mixed temperature throughout the water column, gradually growing in time in response to the surface forcing (Fig. 6a). Early in the experiment, the vertical structure of potential density, $\sigma_{\theta}$, shows an increase of stratification towards the bottom, and the upper $20-\mathrm{m}$ layer shows a weak density gradient for the rest of periods. The cross- and along-shore velocities from the ADCP reveal $\pm 1 \mathrm{~m} \mathrm{~s}^{-1}$ velocity variability with dominating oscillations at a period of approximately 12 hours.

Rotary spectra of the depth-averaged currents measured by the ADCP (Fig. 7a) show a dominant semidiurnal peak, i.e. $\mathrm{M}_{2}$ with $\approx 12.42$-h period. The counterclockwise (CCW) component is slightly, but not significantly, more energetic than the clockwise $(\mathrm{CW})$ components. Figure $7 \mathrm{~b}$ shows the spectra for the cross-shore (black line) and alongshore (red line) components of the current at 3-m height above the seabed (hab). The along-shore component is more energetic at all frequencies. The semidiurnal peak is the
Fig. 5 Time series of a wind speed measurements from cup anemometers at 33-m height above the mean sea level and wind direction from the wind vane at 90-m height, $\mathbf{b}$ significant wave height and peak wave period from a Waverider buoy in the vicinity of the FINO1 and $\mathbf{c}$ wave age estimated from wave bulk parameters using the dispersion relation based on linear wave theory. The vertical dashed lines (grey areas) denote the portion of data used for comparisons between the BL1- and BL2-mounted S1000s

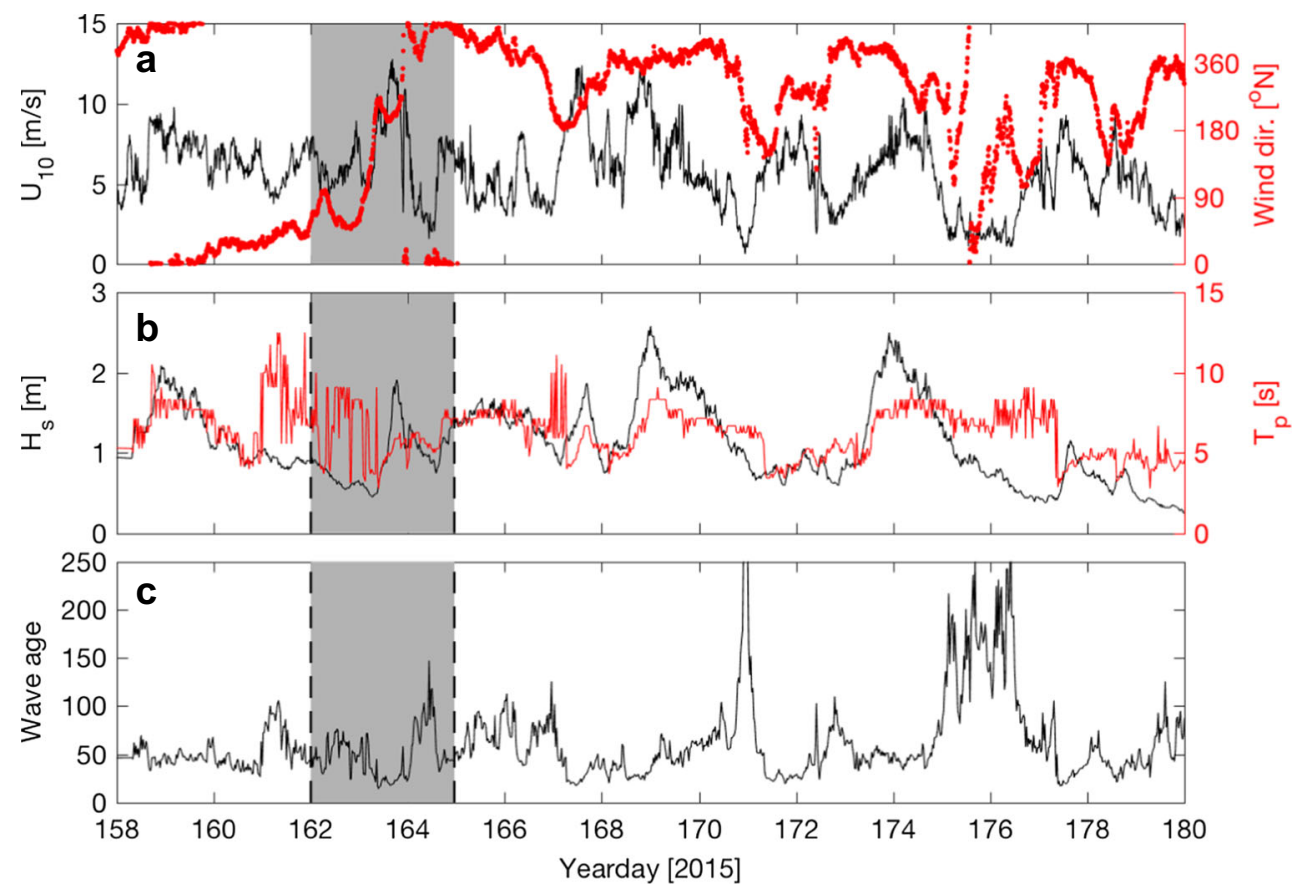


Fig. 6 Time-depth evolution of a temperature, $\mathbf{b}$ density, $\mathbf{c}$ the cross-shore velocity and $\mathbf{d}$ the along-shore velocity component. The temperature data are from the thermistors mounted on the MATS, the OM mooring lines and the FINO1 jacket-mounted sensors. The salinity data are from the OM-mounted CTD loggers at 7.5- and $19.5-\mathrm{m}$ heights above the seabed. The velocity measurements are from the bottom-mounted ADCP a few hundred metres away from FINO1. The vertical dashed lines (grey areas) denote the portion of data used for comparisons between the BL1and BL2-mounted S1000s. The colour in $\mathbf{c}$ and $\mathbf{d}$ have been saturated to $\pm 0.5 \mathrm{~m} \mathrm{~s}^{-1}$ for the sake of better representation

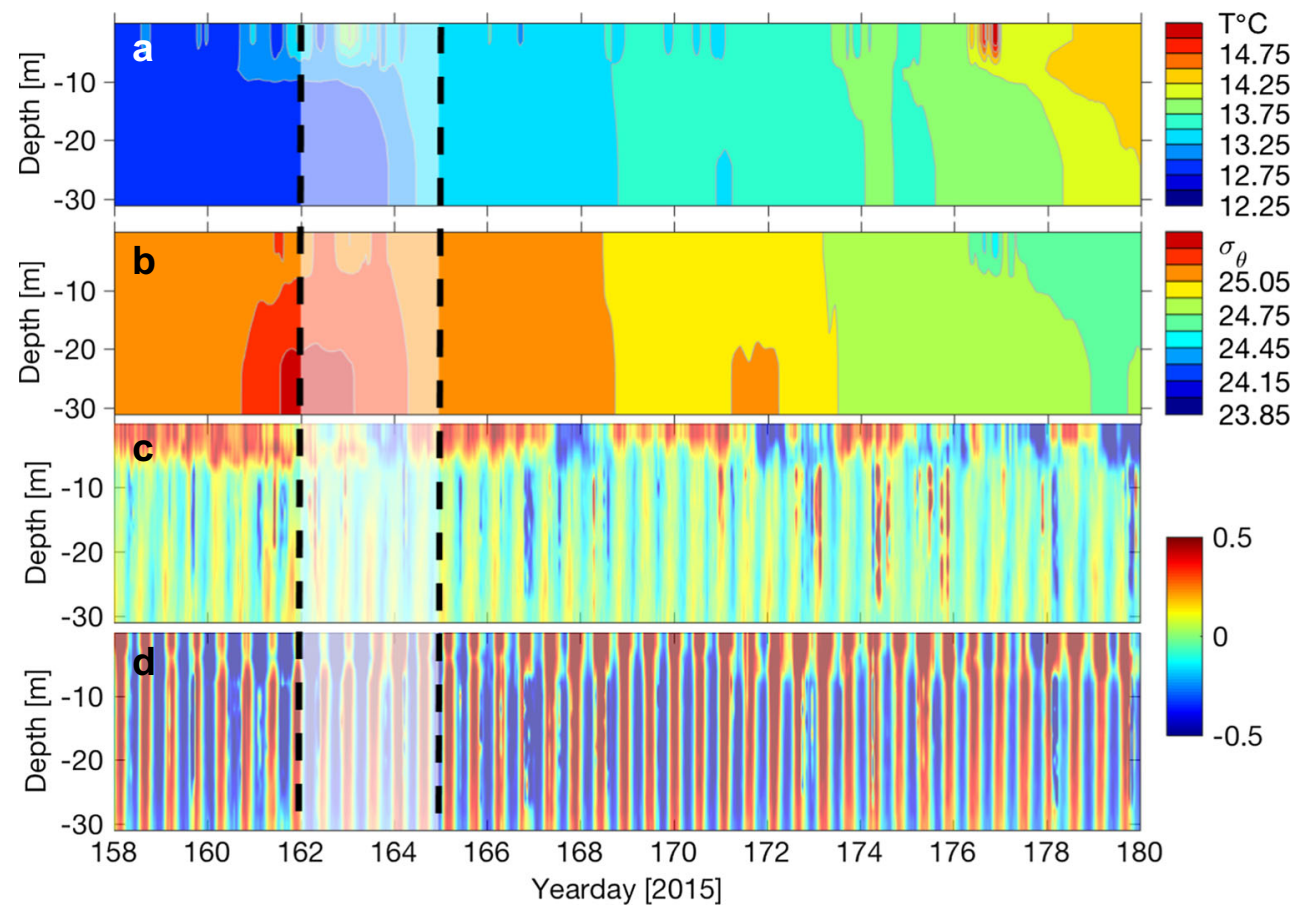

dominant energetic feature, more pronounced for the alongshore component of velocity. Furthermore, sub-harmonics $\mathrm{M}_{4}$ and $\mathrm{M}_{6}$ are also excited.

\subsection{Mean flow variability at BL1 and BL2 locations}

The scatterplots in Fig. 8 show a very good agreement between the current measurements from RCMs at 5.5-m hab (as reference measurements) and S1000s from the closest cell to 5.5-m hab in BL-locations at S1 and S2 (Table 2). Comparisons are shown for along- and cross-shore components separately, and note the change in scales. Regression lines are shown together with the equations and the $95 \%$ confidence bounds. The overall comparisons reveal very good agreement (almost zero-lag correlations) with high values of correlation coefficients, $r$, varying between 0.98 1.0 for all cases. Comparisons in S1 and S2 are based on approximately 3-day period (YD162-YD165) common to BL-mounted S1000s and mooring line mounted RCMs. Figure 8a shows that the regression line for cross-shore velocity does not pass through the origin, and consequently the ratio of velocities slightly changes for different values of $u$. Points outside the error bounds correspond to long waves passing over the $\mathrm{S} 1\left(T_{p}>8 \mathrm{~s}\right.$, not shown).
Fig. 7 a Rotary spectra of hourly barotropic (depth-averaged) currents at the location of ADCP. $\mathbf{b}$ The crossand the along-shore current spectra at 3-m hab. The vertical grey lines show $\mathrm{M}_{2}(\approx 12.42-\mathrm{h})$, $\mathrm{M}_{4}(\approx 6.21-\mathrm{h})$ and $\mathrm{M}_{6}(\approx$ 4.14-h) tidal current constituents
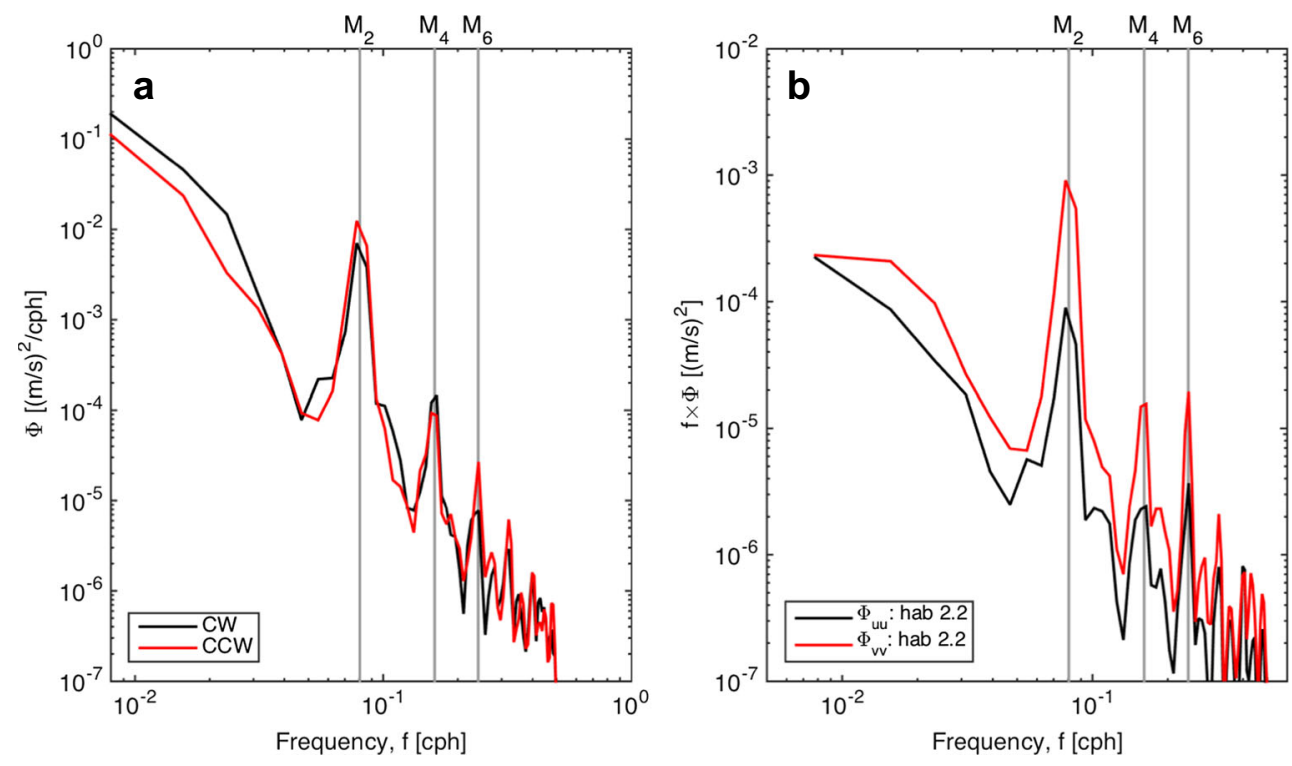

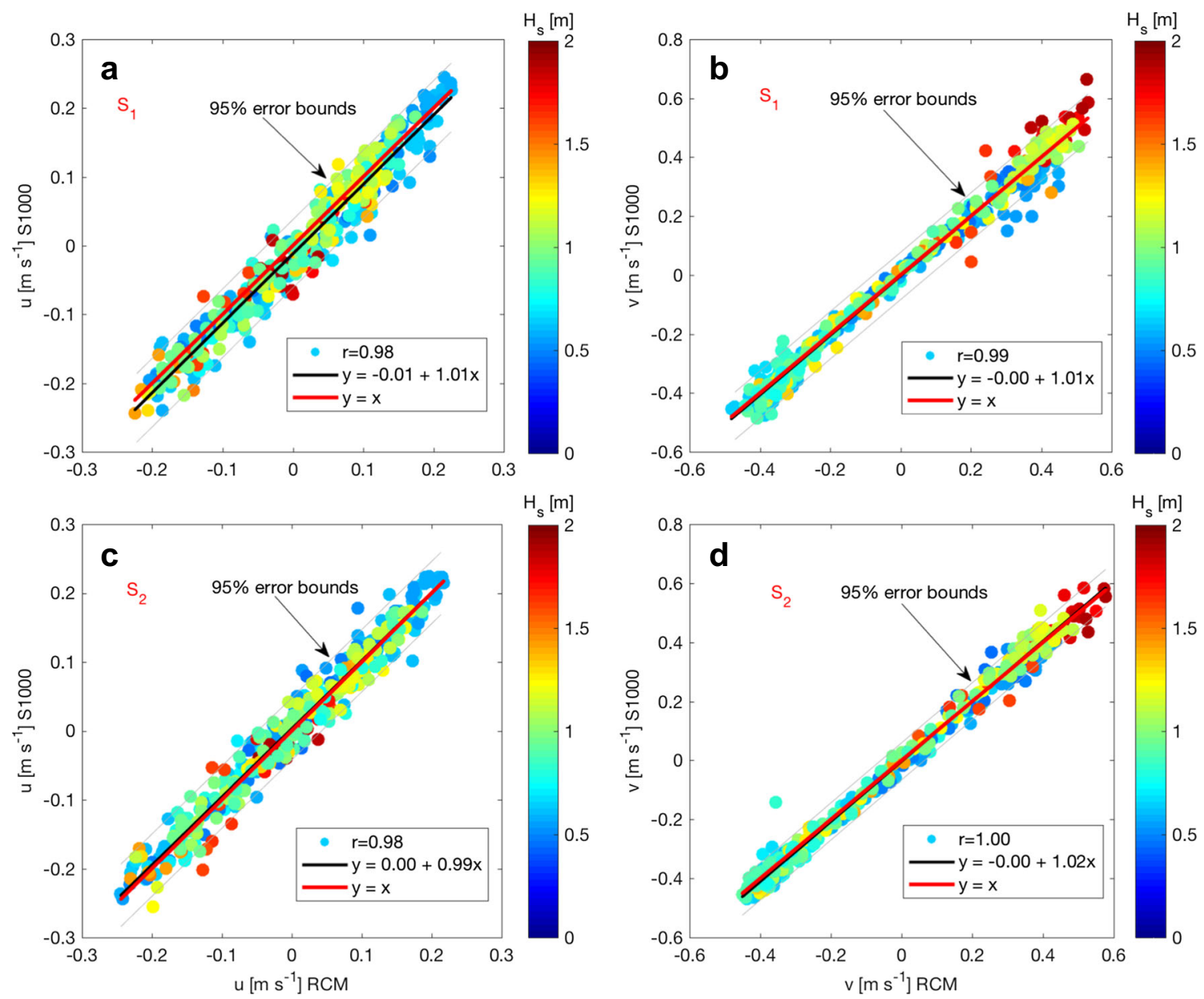

Fig. 8 Scatterplots of 10-min averaged $u$ and $v$ current velocities measured from RCMs at 5.5-m hab and S1000s from the closets cells to 5.5-m hab in a, b S1 and c, $\mathbf{d}$ S2 sites. Markers are colour-coded for $H_{s}$. The black lines are regression lines, error bounds are shown by

grey dashed lines for $95 \%$ level of significance and the red lines are the $45^{\circ}$ for comparison. Data duration at S1 and S2 used in this graph are approximately 3 days (YD162-YD165) for time periods common to BL-mounted S1000s and RCMs

During high-speed events $\left(|v|>0.2 \mathrm{~m} \mathrm{~s}^{-1}\right)$, generally, the scatter in velocity between the co-located RCM and S1000 increases by increasing current amplitude. Comparisons in Fig. 8c at $\mathrm{S} 2$ show a broad scatter in velocity magnitudes when $|u|<0.12 \mathrm{~m} \mathrm{~s}^{-1}$. The vast majority of points outside the error bounds are associated with quasi-long waves with $T_{p} \geq 7 \mathrm{~s}$ and $H_{s} \geq 1 \mathrm{~m}$. For the along-shore velocity component at $\mathrm{S} 2$, most notably the scatter is greatly reduced for a broad values of current speeds with more scatter at high velocity events. Overall comparisons suggest that wind farm would unlikely cause significant differences between the co-located RCMs and S1000s at S1 and S2 in particular for the cross-shore components, while the farm effect is weakly pronounced for the along-shore components when $|v|>0.2 \mathrm{~m} \mathrm{~s}^{-1}$.

The observational current distributions (i.e. histograms) at the depths of RCMs may be approximated, in a statistically significant sense, by the Weibull distribution for both low-wave (LW, i.e. $\left.H_{s}<0.6-\mathrm{m}\right)$ and high-wave (HW, i.e.

$H_{s}>1.1-\mathrm{m}$ ) episodes (Fig. 9). These hourly time-averaged current measurements (see Table 2) provide enough sampling points to conduct such statistical analysis. Given the weak stratification during the period of our experiment, we only look at the shape variability of the current probability distribution at S1 and S2 based on a two-parameter Weibull Probability Density Function (PDF):

$f(s ; \lambda, \kappa)=\frac{\kappa}{\lambda}\left(\frac{s}{\lambda}\right)^{\kappa-1} e^{-(s / \lambda)^{\kappa}}$,

where $\lambda$ and $\kappa$ are scale and shape parameters for this distribution, respectively, and $s$ is a Weibull random number. Table 3 summarises the results of PDF-fitting for the data shown in Fig. 9c, d. The empirical probability distributions characterise the observed PDFs for all episodes. For the LW conditions, the PDF exhibits a larger peakedness with a lower mode (i.e $0.03 \mathrm{~m} \mathrm{~s}^{-1}$ ) at $\mathrm{S} 1$ compared to that of at S2. Furthermore, the distributions suggest a socalled semi-heavy tail for the calm surface forcing conditions. 
Fig. 9 Comparisons between the observational PDFs (i.e. histograms) of horizontal current speeds for the low- and the high-wave events using the RCM data, and the fitted Weibull distributions (red curves) at $5.5-\mathrm{m}$ hab at $\mathrm{S} 1$ and $\mathrm{S} 2$ experimental sites
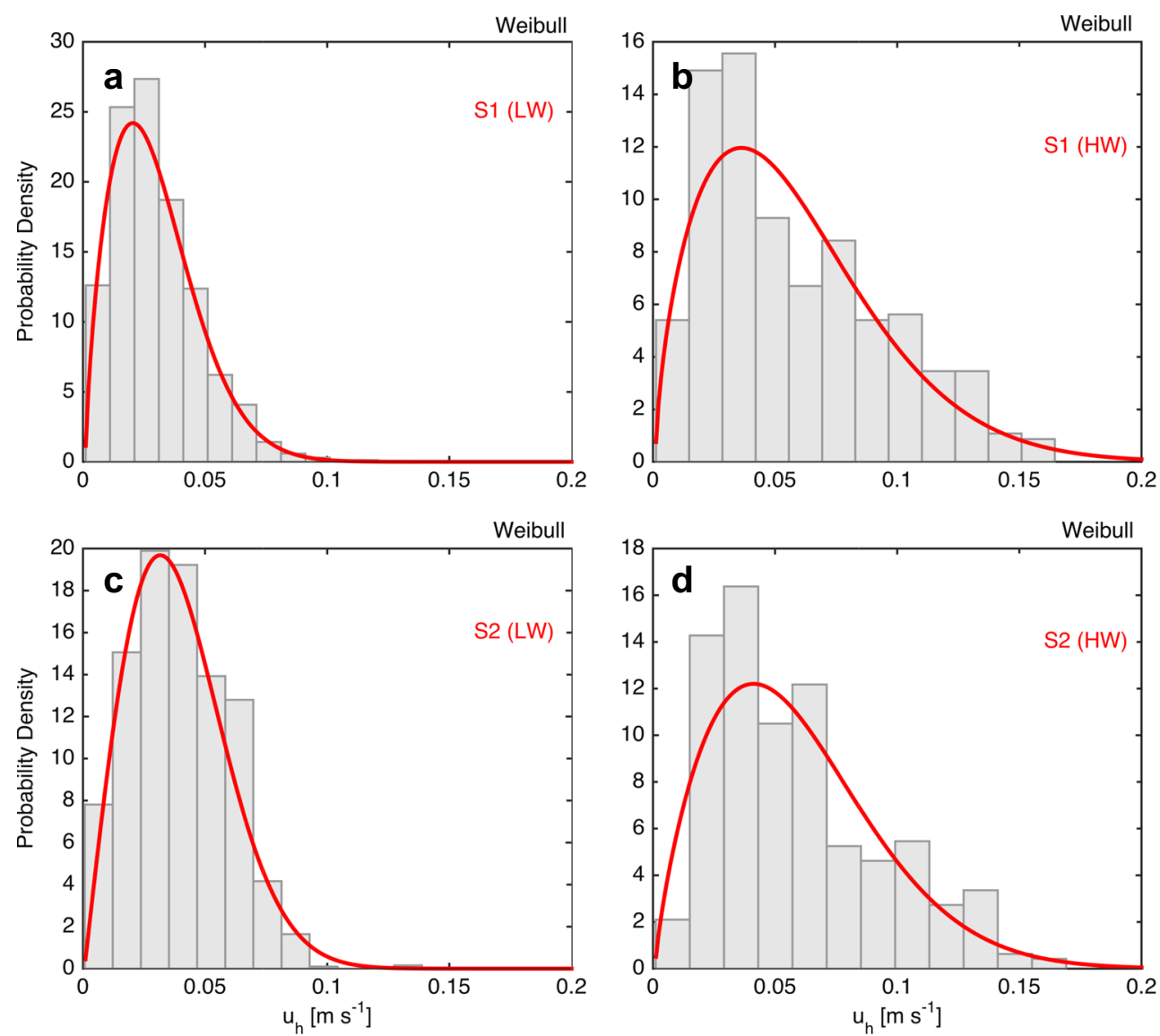

During strong wind events, two PDFs at S1 and S2 have approximately the same peakedness and (positive) skewness. The distributions at S1 have slightly lower mode than those at S2 (i.e $0.04 \mathrm{~m} \mathrm{~s}^{-1}$ ), and both PDFs at S1 and S2 during strong surface forcing conditions exhibit light-tail distributions.

Figure 10 illustrates histograms for the RCM-based cross- and along-shore velocity components at S1 and S2. The shapes of all histograms are Gaussian-like, in particular for the along-shore components, and generally show skewnesses towards the positive values of velocities. The cross-shore variance at $\mathrm{S} 2$ is slightly higher than the one at $\mathrm{S} 1$, while no statistically significant differences have been observed for the along-shore velocity statistics, and the frequency distribution of the along-shore current is rather symmetric around the mean value. The histograms of velocity directions at two sites are also symmetric around the most frequent angle of $180^{\circ}$ with approximately normal distributions (not shown).

Figure 11 summarises the contour plots of horizontal and vertical velocities measured from the BL-mounted S1000s (in averaged-mode based on four beams) between YD162 and YD165. The cross-shore velocities (Fig. 11a, b) have weaker, by approximately a factor of 2, amplitudes than the along-shore velocities (Fig. 11c, d) and the tidal currents dominate in the along-shore direction at S1. Generally, the horizontal and vertical velocity distributions are similar at sites S1 and S2. At heights well below any possibility of instrument's side-lobe contaminations, the magnitudes of cross-shore current profiles are high near the bed and

Table 3 Summary of the statistical parameters for the Weibull distribution, the cross- and along-shore currents, and horizontal current speed, $u_{h}$, for the data reported in Fig. 9

\begin{tabular}{|c|c|c|c|c|c|c|c|}
\hline Site & Sea-state & & $\kappa$ & $\lambda$ & $u$ & $v$ & $u_{h}=\sqrt{u^{2}+v^{2}}$ \\
\hline \multirow[t]{2}{*}{$\mathrm{S} 1$} & Calm & $H_{s}<0.6$ & 0.03 & 1.71 & 0.01 & 0.0 & 0.03 \\
\hline & Wavy & $H_{s}>1.1$ & 0.06 & 1.62 & -0.02 & 0.02 & 0.06 \\
\hline \multirow[t]{2}{*}{$\mathrm{S} 2$} & Calm & $H_{s}<0.6$ & 0.04 & 2.01 & 0.03 & 0.0 & 0.04 \\
\hline & Wavy & $H_{s}>1.1$ & 0.07 & 1.76 & 0.01 & 0.03 & 0.06 \\
\hline
\end{tabular}


Fig. 10 Comparisons between the observational PDFs of the cross- and the along-shore velocities from the RCM-based velocities, and fitted normal distributions (red curves) at 5.5-m hab at $\mathrm{S} 1$ and $\mathrm{S} 2$ experimental sites
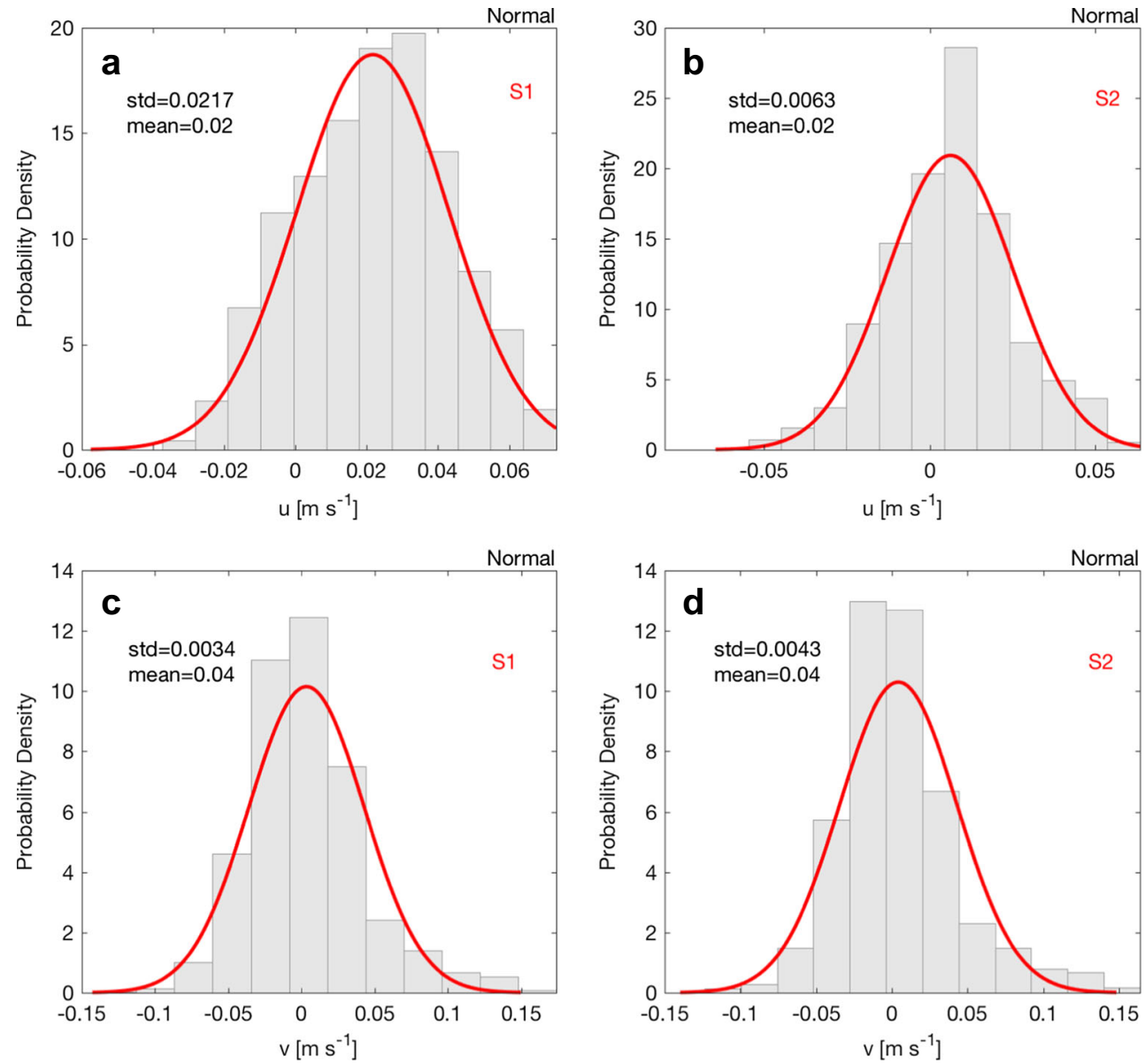

decrease towards the surface, whereas the magnitudes of along-shore profiles are larger at the surface and slowly decrease towards the bed. Figure 11e, f show detailed structure of vertical velocity profiles (measured from four beams). Strong vertical banding in the measured $\bar{w}$ profiles suggests larger response of ocean vertical motions to the forcing variability at $\mathrm{S} 1$ versus $\mathrm{S} 2$ in particular at interior layers (e.g. YD163-YD163.5) when internal waves or wave-bottom interaction (e.g. bottom friction) might be important forcing mechanisms. In addition to some variability expected because of the spatial separation between BL1 and BL2, instrument tilt error, and errors induced by applying pitch- and roll-correction algorithm, we can also expect wind farm wake disturbances.

\subsection{Average profiles}

Figure 12 demonstrates ensemble-averaged (horizontal) current profiles during YD162-YD165 over calm and wavy surface forcing conditions in S1 and S2. A total number of 52 and 85 10-min observations are used to compute the mean profiles for the weak and strong wind and wave forcing conditions, respectively. Velocity profiles during
LW and HW episodes (Table 3) at S1 illustrate a sharp increase near the sea surface, as expected. While qualitative shapes of profiles at BL1 location remain similar for two ensembles, the magnitudes of velocities near the sea surface are larger than those during the calm periods as a result of the response of uppermost currents to variable surface forcing conditions. The current vertical structure at S2 is generally surface-intensified with smaller values, within the shown confidence intervals, near the sea surface than the similar measurements made at $\mathrm{S} 1$. Three days of observations used for this analysis are not, however, sufficient to describe the role of the wind farm in modulation of ocean current profiles. Additionally, the computed ensembles generally exhibit tendency towards near-surface overestimation due to the potential side-lobe interferences of acoustic measurements very close to the air-sea boundary.

\subsection{Vertical velocity spectra}

Figure 13 shows the frequency spectra of vertical velocity fluctuations, $w^{\prime}$, from the upward pointing beam (i.e. $b_{5}$ for firmly levelled S1000) over heights below 24-m above the seabed. Variation in the vertical evolutions of energy 
Fig. 11 Contour plots of the mean cross-shore $(\bar{u})$, along-shore $(\bar{v})$, and vertical $(\bar{w})$ velocities measured from $\mathbf{a}, \mathbf{c}, \mathbf{e}$ the BL1-mounted S1000 and b, d, $\mathbf{f}$ the BL2-mounted $\mathrm{S} 1000$ sampled in averaged mode (i.e. 2-min averages every 10 -min)
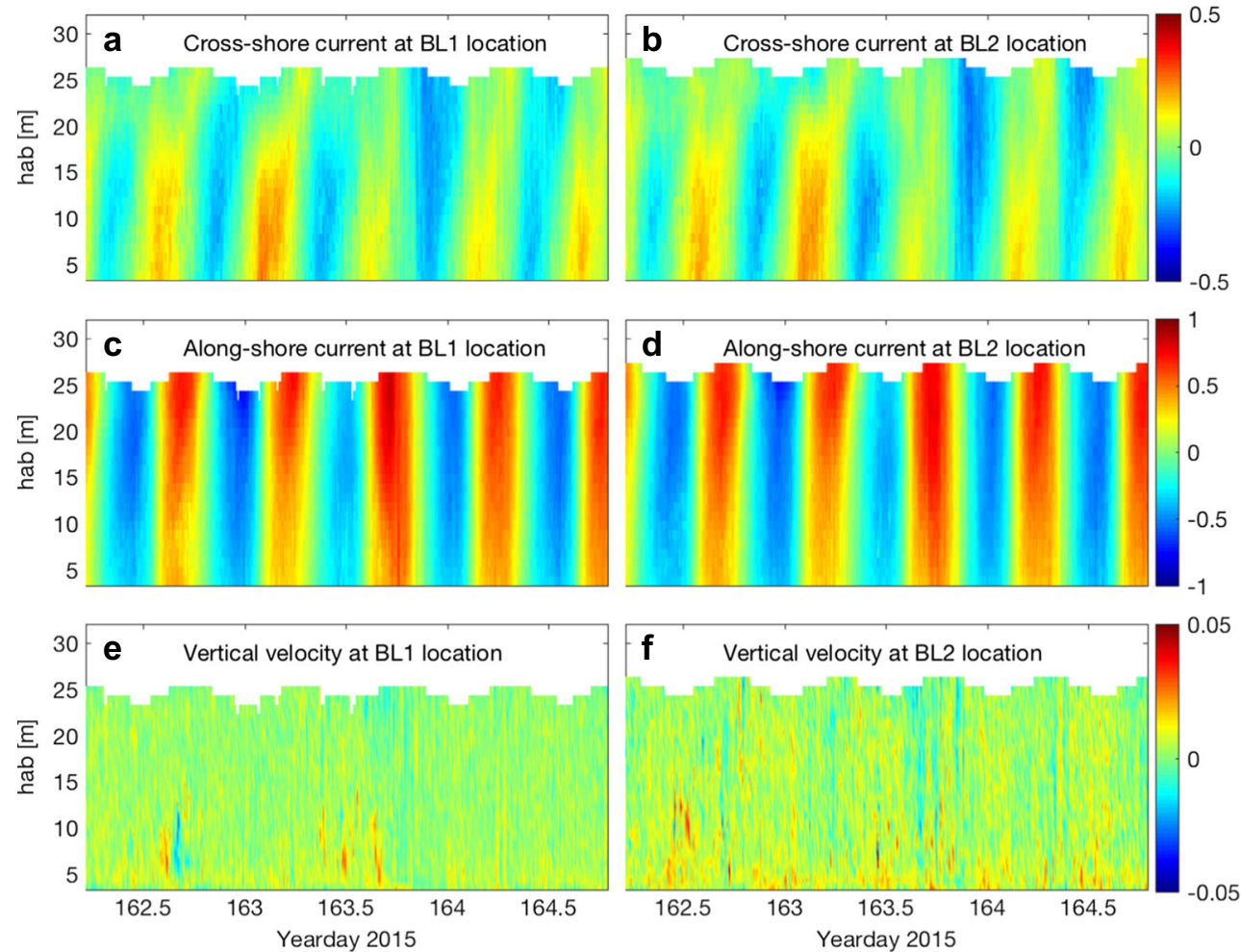

distribution is clearly linked to the rapid attenuation of wave signals away from the air-sea interface. In general, all vertical velocity spectra at different vertical levels exhibit a wave band at which its bandwidth has tendency to decrease exponentially at higher frequencies towards the seabed. There exists further a systematic shift in energy peaks, as expected,
Fig. 12 Time-averaged horizontal velocities measured during YD162-YD165 for low-wave (LW) and high-wave (HW) conditions from the BL1and BL2-mounted S1000s (see Table 3)
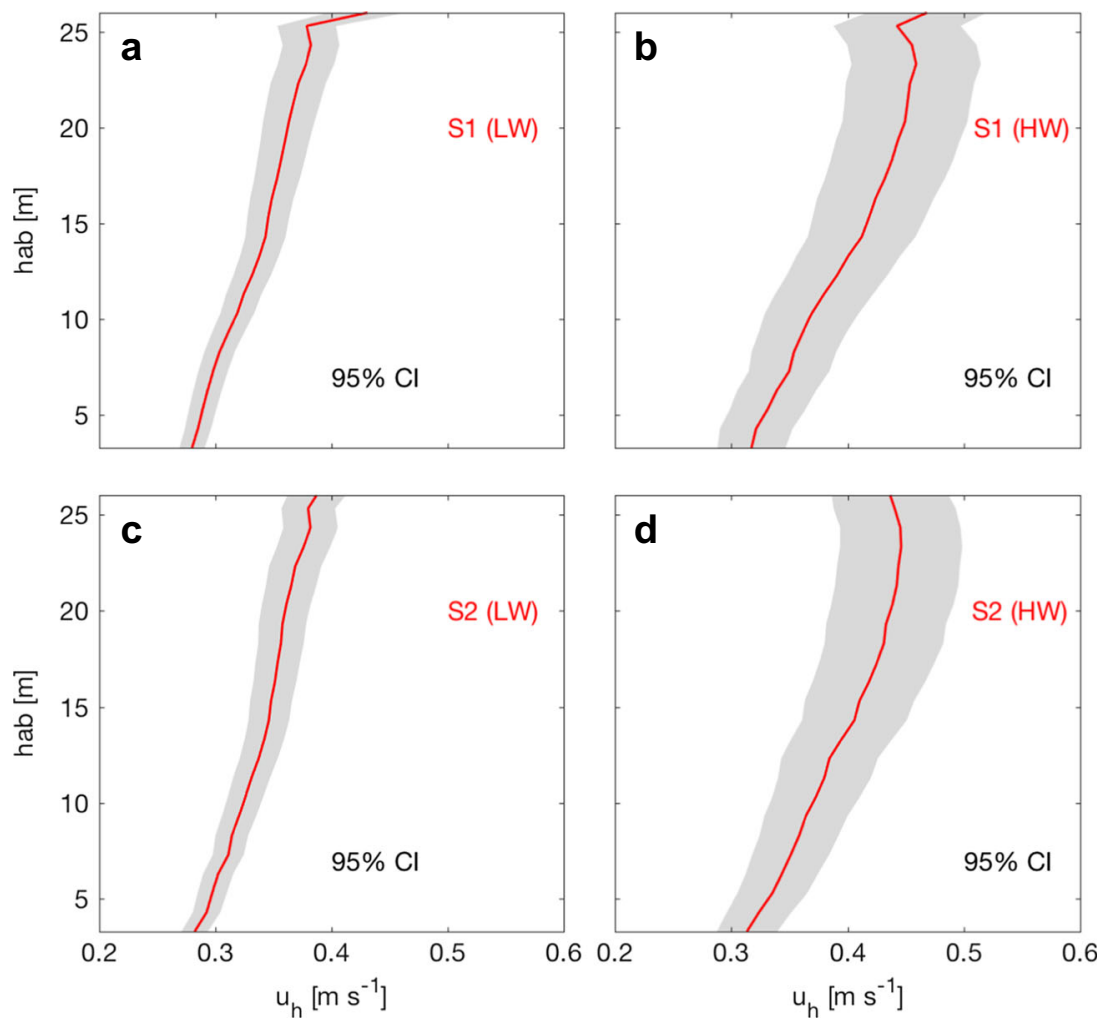


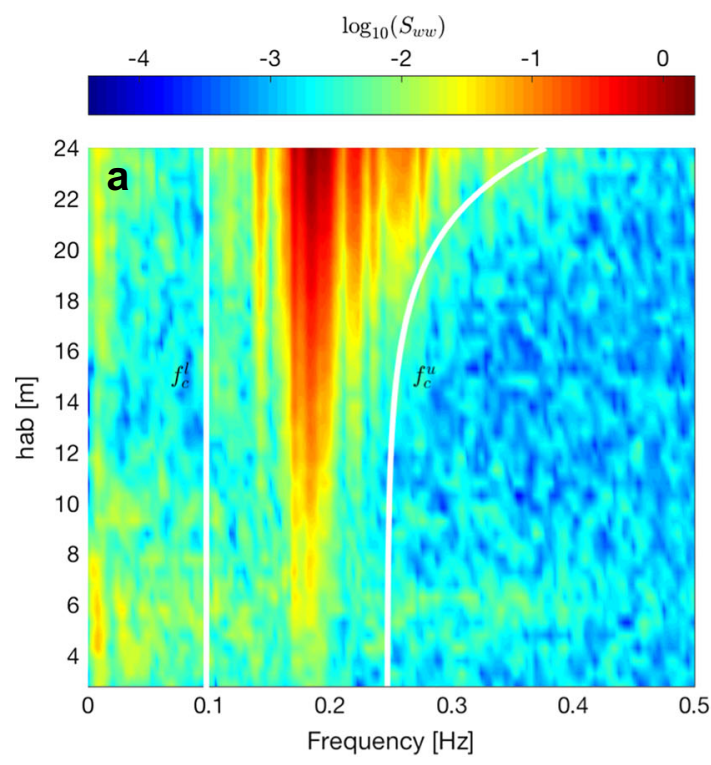

Fig. 13 a Frequency spectra of vertical velocity fluctuations (variances are colour-coded in $\log _{10}$ scale) at heights below 24-m above the seabed for an arbitrary burst from the BL1-mounted S1000. b The

towards lower frequencies as a function of depth. Energy elevation at below-wave frequencies is a result of the dominant tidal motions. Vertical profiles of the lower, $f_{c}^{l}$, and the upper, $f_{c}^{u}$, frequency bounds of the wave-affected subrange (white lines) are defied as

$\frac{f_{c}^{l}}{f_{p}}=0.5 ;$ and $\frac{f_{c}^{u}}{f_{p}}=a+b \exp \left(-\frac{|z|}{\lambda_{w}}\right)$,

where for our datasets, values of $a=1.05, b=1$, and $\lambda_{w}=$ $1 /\left(2 k_{p}\right)$ are set by checking manually all velocity spectra.

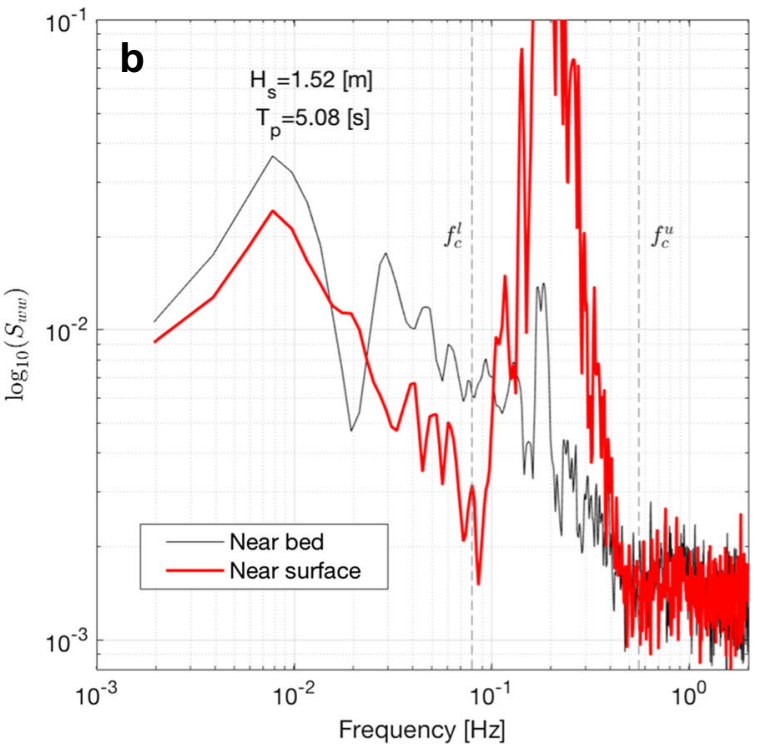

corresponding spectra for the uppermost (red) and the lowermost (black) cells. The vertical lines indicate the lower and upper frequency bounds for the wave band

Here, $z$ is the distance from the sea surface and $k_{p}$ denotes the wavenumber at the wave peak frequency calculated from the dispersion relation for linear waves. The power spectra of $w^{\prime}$ for the uppermost and lowermost cells are characterised by high energy content across a broad range of wave-affected frequencies accompanied by flat and noisy energy spectra above the wave band. Both vertical velocity spectra have a peak with different amplitude at $f_{p}$, which is attributed to the waves, and the uppermost spectrum flattens out at frequencies $>f_{p}$ and below $f_{c}^{u}$ while the lowermost
Fig. 14 One-sided cross-spectral density of $u^{\prime}$ and $w^{\prime}$ for the uppermost cell below the sea surface. Vertical dashed lines denote $f_{c}^{l}$ and $f_{c}^{u}$ calculated from Eq. 30, and the black line displays the wave peak frequency for an arbitrary burst of S1000 at BL1

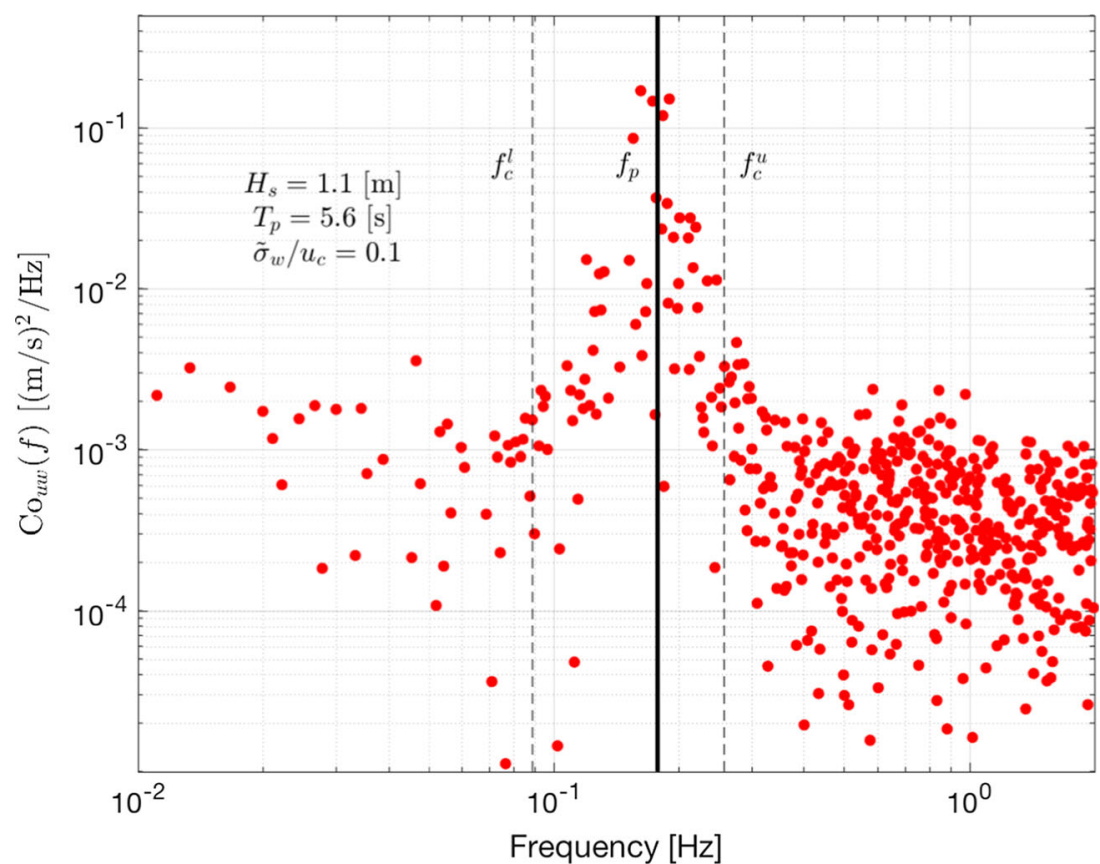


spectrum still continues to cascade down. The slope of the surface spectrum does not show a clear characteristic $-5 / 3$ power-law beyond the wave band, while a narrow-band inertial subrange (where TKE cascades downward from larger to smaller scales) emerges closer to the seabed.

\subsection{Reynolds stresses}

An example of cospectrum, $\mathrm{Co}_{u w}(f)$, for a sample nearsurface burst measured from the BL1-mounted S1000 is shown in Fig. 14. As expected, there is a significant contribution to the cospectrum at frequencies between $f_{c}^{l}$ and $f_{c}^{u}$ from surface waves. The observed cospectrum is highly scattered particularly at high frequencies, and is little affected by wave orbital motions at frequencies higher than $f_{c}^{u}$ because the rms wave orbital velocity is smaller than $u_{c}$ for this burst. Furthermore, wave-induced variances decay at low frequencies below $f_{c}^{l}$. This suggests that the advection of turbulence for this burst at frequencies lower/higher than the wave band is mainly due to the mean current. Note that this burst is for the uppermost cell, close to the sea surface, and the wave bandwidth near the sea surface is much wider than those at deeper levels, with large effects on the covariances near the peak wave frequency. By taking distance from the air-sea interface, the wave peak bandwidth becomes narrower and the wave effects on the integral of (co)spectrum reduces substantially (see also Fig. 13).

The wave advection effect on observed RSs is summarised in Fig. 15a that illustrates the time-depth variation of the ratio between the rms wave orbital velocities, $\tilde{\sigma}_{w}$, and the mean current speeds measured from the BL1-mounted $\mathrm{S} 1000$. The values of $\tilde{\sigma}_{w}$ are estimated using the integration of the spectral densities of the observed fluctuating velocities within the wave frequency range $\left[f_{c}^{l}, f_{c}^{u}\right]$. While the spatio-temporal variation of $R=\tilde{\sigma}_{w} / u_{c}$ near the sea surface is controlled by the wave- and the wind-related processes (see Fig. 5a), the wave-advection at energycontaining scales and at scales well above $f_{p}$ play little
Fig. 15 a Time-depth evolution of the ratio between rms wave orbital velocity and mean current speed, $R=\tilde{\sigma}_{w} / u_{c}$. $\mathbf{b}$ Vertical profiles of the observed, ${\overline{u^{\prime} w^{\prime}}}^{o b s}$ and the modelled, ${\overline{u^{\prime} w^{\prime}}}^{\text {model }}$, cross-shore RSs. c Vertical profiles of the observed and the modelled friction velocities. d Vertical distribution of the observed TKE, $q_{o b s}^{2}$ and the modelled TKE, $q_{\text {model }}^{2}$
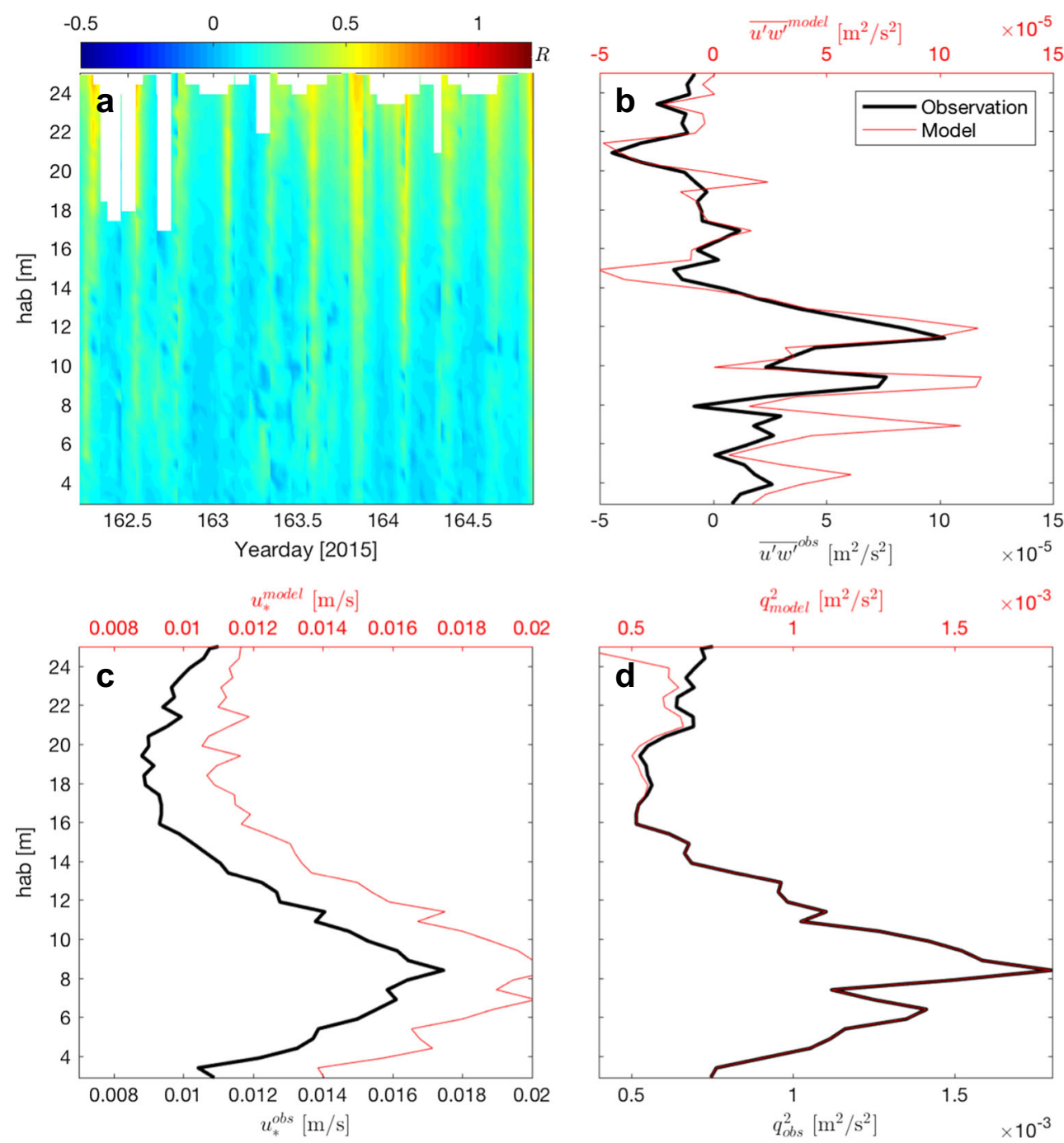
role in rearrangement of turbulence energy at those frequencies. The vertical profile of time-averaged $\overline{u^{\prime} w^{\prime}}$ obs over YD162-YD165 is compared with the modelled cross-shore $\mathrm{RS},{\overline{u^{\prime} w^{\prime}}}^{\text {model }}$, calculated from information of the spectral densities at low frequencies $\left(\ll f_{p}\right)$ where the observed cospectrum is almost flat, Fig. 15b. In this figure, the constant spectral density averaged over very low frequencies, $\mathrm{Co}_{u w}^{\text {lf }}$, is multiplied by an exponentially decaying factor as a function of $R, f, u_{c}$ and $f_{p}$, i.e. Eq. 27. The modelled and observed RSs are highly correlated, suggesting less waveinduced covariances at low frequencies (i.e. $f<f_{c}^{l}$ ) and therefore underestimation of ${\overline{u^{\prime} w^{\prime}}}^{o b s}$ may be explained by removing the turbulence energy from the portion of the cospectrum within the wave-affected frequency band. To diagnose the wave advection effects at low frequencies, we compare the observed time-averaged friction velocity and TKE profiles with the modelled ones. The low frequency portion of the observed frequency spectra and cospectra are less affected by wave advection and the turbulence quantities are in close agreement with the model predictions.

Reynolds stresses in the cross- and along-shore directions are computed using the VM between YD162 and YD165 for data collected from the BL1-mounted S1000, Fig. 16. During the strong surface wind and wave forcing episodes, the surface-generated stresses are extended downward through the water column and merged by the bottom-intensified stresses which vary typically over the semidiurnal $\mathrm{M}_{2}$ tidal cycle. Applying the wave-correction algorithm removes substantially the large wave-induced variances from the estimated stresses (Fig. 16a, c) and reduces their magnitudes by nearly one order of magnitude (Fig. 16b, d). The wave-corrected along-shore stress, $\left(\overline{v^{\prime} w^{\prime}}\right)_{c}$, reveals stronger response to the major wind event (i.e. around YD163.5) by spanning the turbulence throughout the water column compared with the response of the cross-shore component to the surface variability, $\left(\overline{u^{\prime} w^{\prime}}\right)_{c}$, Fig. 16b. Some asymmetric features are clearly observed in the vertical structures of $\left(\overline{u^{\prime} w^{\prime}}\right)_{c}$ and $\left(\overline{v^{\prime} w^{\prime}}\right)_{c}$ that may be explained by the effects of weak stratification, interactions between surface and bottom boundary layers and poor stationarity of velocity field (in particular for the horizontal velocity components). The magnitudes of these burst-type events, separated by nearly quiescent periods, are closely connected to different phases of tidal cycle for mainly lower part of RS profiles.

We also check the accuracy of the measured RSs in Fig. 17 by comparing the values of $u_{* w}$ predicted by the drag law using wind speed (Large and Pond 1981) to those obtained from the uncorrected and wave-corrected time series of RS at the uppermost bin below the sea surface. Due to the S1000 side-lobe contamination at the air-sea interface, the Reynolds stresses in this graph are taken for depths about one meter below the sea surface. Computed values for RSs are then filtered in time to reduce the noise using a four-point running average filter. It is observed that the wave-induced disturbances lead to an order of magnitude overestimation in the observed friction velocities relative to those calculated from the drag law (in the bulk sense), and applying the correction procedure successfully
Fig. 16 Depth-time series of a, b the uncorrected, $\left(\overline{u^{\prime} w^{\prime}}\right)_{r}$, and the wave-corrected, $\left(\overline{u^{\prime} w^{\prime}}\right)_{c}$, cross-shore RSs measured from the BL1-mounted S1000 and c, d the uncorrected, $\left(\overline{v^{\prime} w^{\prime}}\right)_{r}$, and corrected, $\left(\overline{v^{\prime} w^{\prime}}\right)_{c}$, along-shore RSs measured from the BL2-mounted S1000
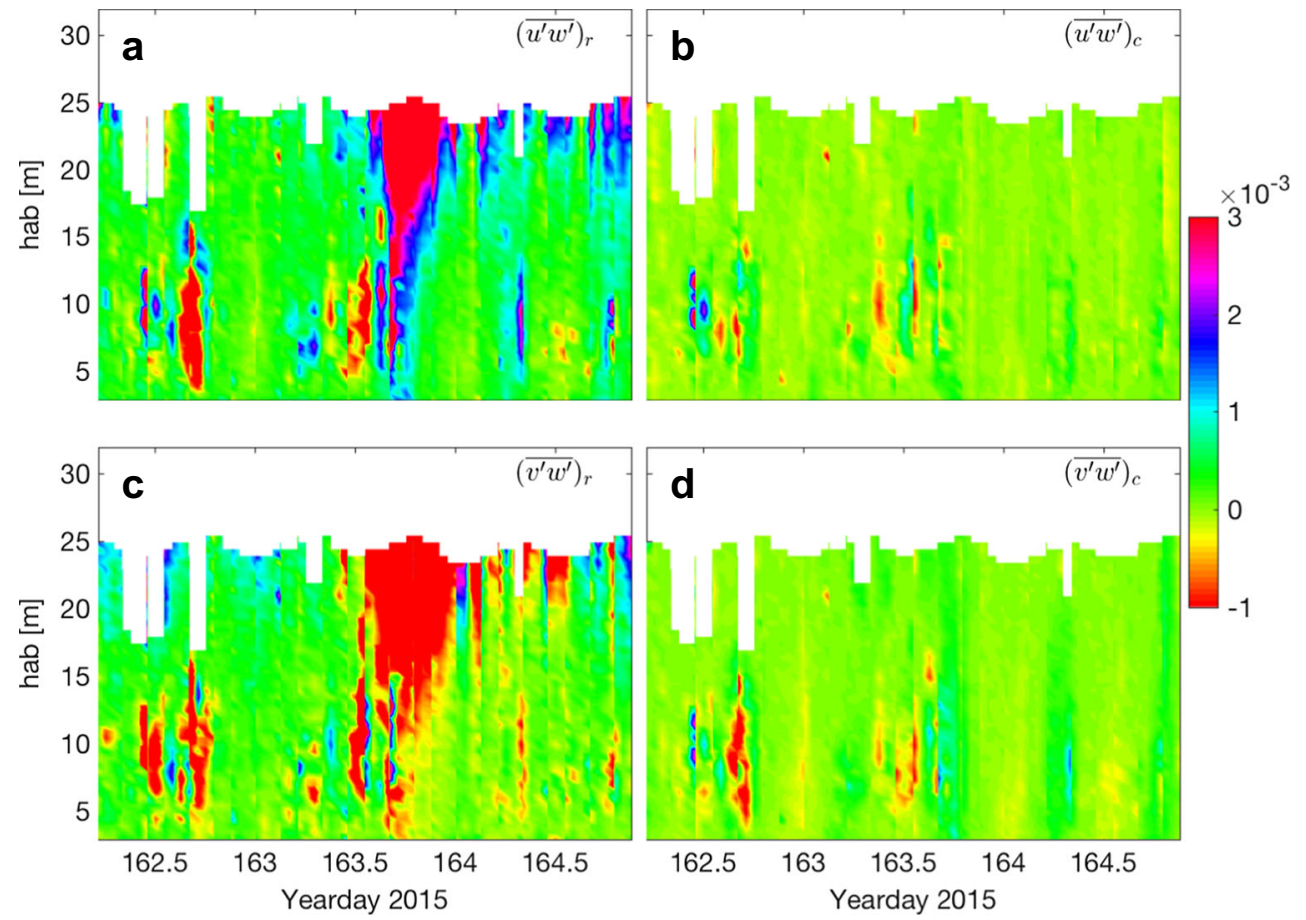

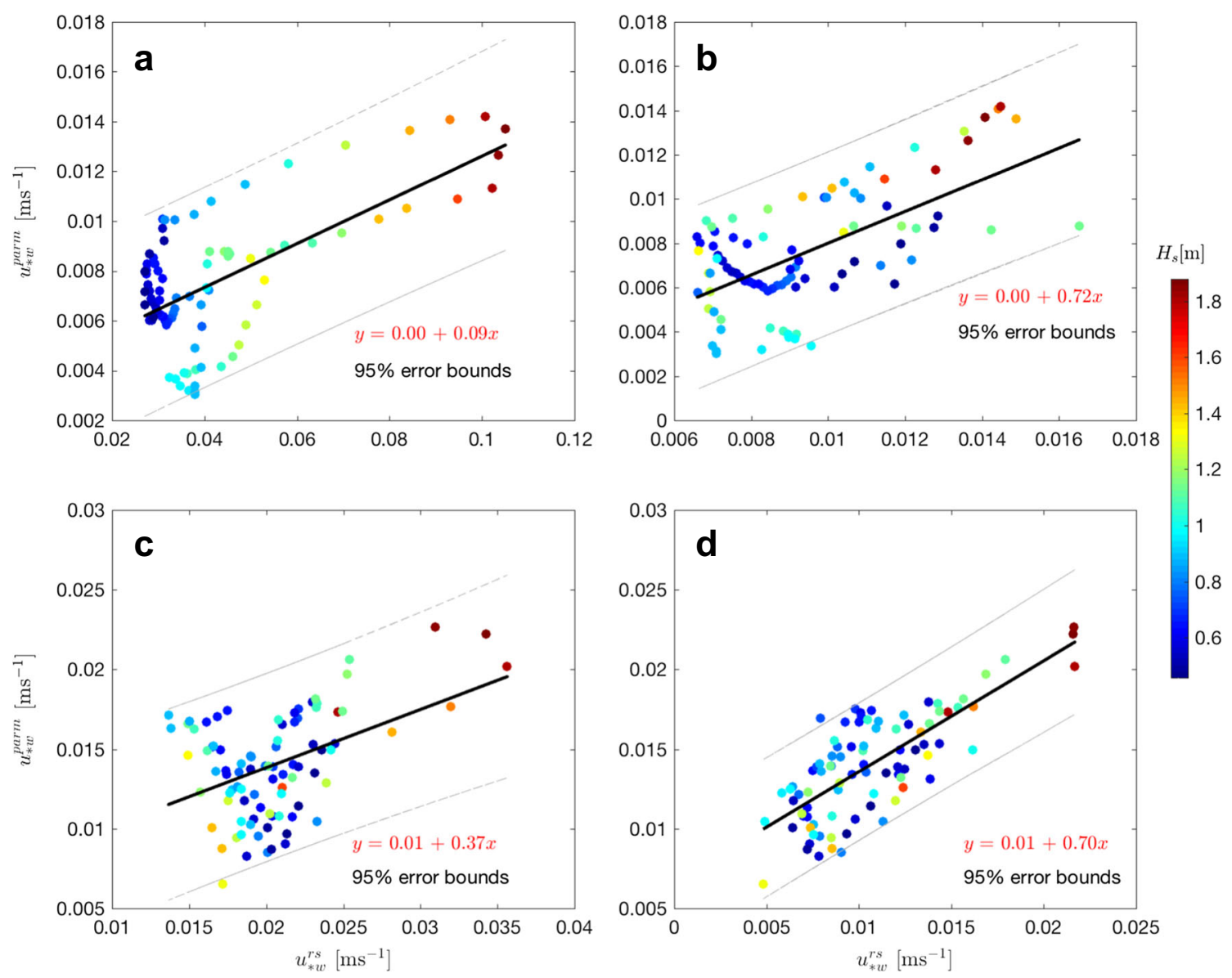

Fig. 17 The scatterplot of the sea surface and bottom friction velocities computed using drag laws (Large and Pond 1981; Nielsen 1992) compared to the magnitude of the $\mathbf{a}, \mathbf{c}$ uncorrected and $\mathbf{b}, \mathbf{d}$ corrected observed friction velocities. The colour of markers show the correlation

between the wave-induced bias and significant wave height in metres. Data are for duration between YD162 and YD165 collected from the BL1-mounted S1000

removes the majority of the wave-induced variances from RS estimates. Both uncorrected and wave-corrected friction velocities also exhibit a strong increase and departure from the drag law during YD164-YD164.5 due to the Doppler phase noise in the uppermost S1000's sampling volume and side-lobe contaminations caused by strong surface forcing events (colour of markers are scaled by $H_{s}$, see also Fig. 11e). Figure 17c and d illustrate comparisons between the near bottom friction velocities measured by the lowermost RS time series and those estimated from the drag law $u_{* b}=\sqrt{\tau_{b} / \rho_{w}}$, where $\tau_{b}=\left(\tau_{b}^{x}, \tau_{b}^{y}\right)=$ $C_{d} u_{b}\left|u_{b}\right|$ is the bed stress, and $C_{d}=\left(C_{d}^{x}, C_{d}^{y}\right)$ denotes the drag coefficients depending on the seabed composition, height above the seabed, waves and the tidal current speeds. We calculate $C_{d}$ using the Chézy formulation for sediment particle size of $0.02 \mathrm{~mm}$ in the absence of wave effect (Nielsen 1992). The comparisons show much better agreement between the observed and the bulk friction velocities at the bottom boundary layer when the wavecorrected RSs are used to estimate $u_{* b}$. The scatters in Fig. $17 \mathrm{~d}$

is partly explained by the effect of excluded turbulent spectral densities at frequencies beyond $f_{c}^{l}$, and in part due to other sources of uncertainty such as form drag effects.

Figure 18 shows profiles of time-averaged (wave- and tilt-corrected) along-shore drag coefficient, $C_{d}^{y}$, over wavy $\left(H_{S}>1.1 \mathrm{~m}\right)$ and calm $\left(H_{S}<0.6 \mathrm{~m}\right)$ sea-state conditions (Fig. 18a, b), and flood and ebb tidal conditions (Fig. 18c, d) for the data collected from the BL1- and BL2-mounted S1000s during YD162-YD165. We estimate 95\% confidence interval using $t$ distribution of the observed time series at each vertical level. There is evidence of strong drag variability throughout the water column for the LW case at both measuring locations (Fig. 18a). Meanwhile, the presence of wind farm has modulated the magnitude of the observed $C_{d}^{y}$ at heights near the sea surface during the LW events with less variability very close to the seabed. The implications for the local and non-local interactions between waves and turbulence can be assessed from the noticeable level of uncertainty (noise) observed in the reported measurements (e.g. residual wave-induced 
Fig. 18 Time-averaged vertical profiles of $C_{d}^{y}=\overline{v^{\prime} w^{\prime}} / u_{c}^{2}$ during YD162-YD165 at S1 and S2 over a the LW conditions, $\mathbf{b}$ the HW sea-state conditions, $\mathbf{c}$ ebb tides and $\mathbf{d}$ flood tides. The shaded areas denote $95 \%$ confidence interval calculated from $t$ distribution of $C_{d}^{y}$ time series at each height above the seabed
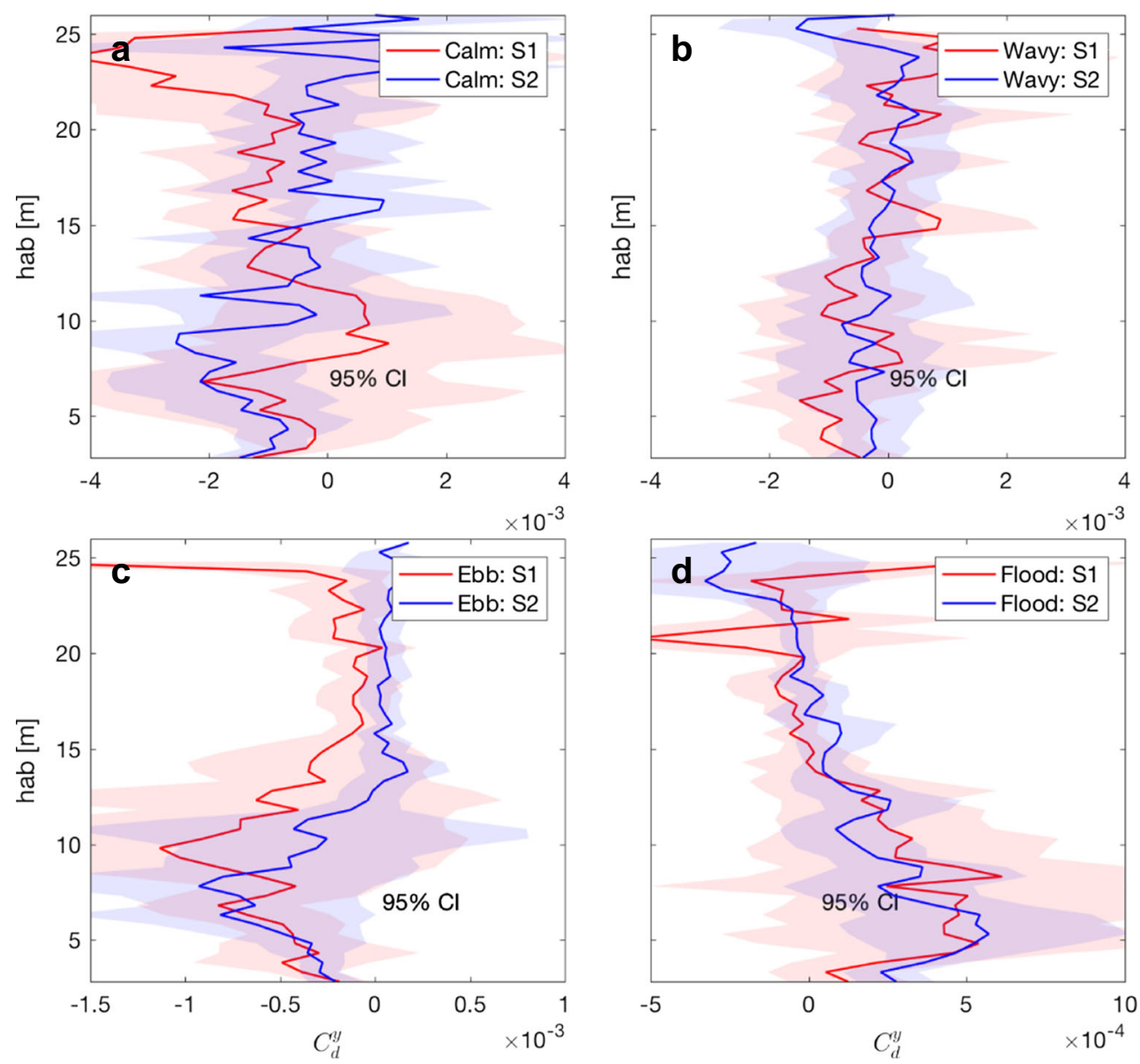

variances due to an unreliable detection of the below-wave cutoff frequency). The vertical series of the time-averaged $C_{d}^{y}$ during the HW conditions show that effects of farm wake cause a weak modulation in the vertical distribution of RSs (and $C_{d}^{y}$ ) throughout the water column. Figure 18c and $d$ give another impression for the vertical variability of time-averaged $C_{d}^{y}$ over the tidal cycles. The asymmetry of $C_{d}^{y}$ during ebb and flood tides at two sites are clearly pronounced at heights between 5 and $15 \mathrm{~m}$ above the seabed (presumably due to the tidal straining). During the ebb tides, both time-averaged drag coefficient profiles at $\mathrm{S} 1$ and $\mathrm{S} 2$ are generally negative, except $C_{d}^{y}$ at $\mathrm{S} 2$ which is positive for the upper half water column. On flood tides, the $C_{d}^{y}$ profiles at two sites show comparable vertical patterns in the absence of influences from the farm-induced wake at $\mathrm{S} 2$.

\subsection{TKE, $P_{s}$, ATKE and $I_{u}$}

The wave- and tilt-corrected TKE, $\mathrm{P}_{s}$, ATKE and $\mathrm{I}_{u}$ are shown in Fig. 19 for BL1- and BL2-mounted S1000s. All turbulence metrics measured in these locations reveal similar characteristics in spite of approximately $3 \mathrm{~km}$ of separation distance. The high values for the TKE and the shear production, $\mathrm{P}_{s}$, are found near the surface and in the lower layers due to the strength of surface forcing, bottom friction and strong tidal mixing (Fig. 19a, b). However, it is not clear, from these figures whether the observed variations are from superimposition by the wind wake behind the Alpha Ventus or because of the spatial separation between measuring platforms. By an abrupt increase in the wind speed during YD163.5-YD164 (Fig. 5b), the surface layers at $\mathrm{S} 1$ show strong tendency towards being more isotropic. In contrast, the counterpart measurements in S2 exhibit more anisotropic features in this period at the uppermost layers as a result of farm wake effects on modulation of wind and wave fields. The anisotropic variances (originated from weak stratification, wave-bottom interaction and farminduced wake effects) make the flow highly unbalanced with respect to the momentum, energy and vorticity fields (Fig. 19c, d). Figure 19e, f shows the spatio-temporal evolution of the cross-shore component of turbulence intensity, $I_{u}$, in $\mathrm{S} 1$ and $\mathrm{S} 2$, respectively. The values of turbulence intensities are consistent with the variations of TKE and $\mathrm{P}_{s}$ with nearly the same temporal/spatial characteristics. Furthermore, increased magnitudes of $\mathrm{I}_{u}$ when currents are relatively weak (below $0.1 \mathrm{~m} \mathrm{~s}^{-1}$ near the surface), are inconsistent with the relationship given by Eq. 28. This suggests that the non-local variances from farm wake and waves 
Fig. 19 Depth-time series of different turbulence parameters at $\mathrm{S} 1$ and $\mathrm{S} 2$ from the wave-corrected data during YD162-YD165. Contour plots of $\mathbf{a}, \mathbf{b}$ TKE from BL1- and BL2-mounted S1000s, respectively; $\mathbf{c}, \mathbf{d} \mathrm{P}_{S}$ at $\mathrm{S} 1$ and S2; e, f ATKE at S1 and S2 sites; and $(\mathbf{g}, \mathbf{h}) \mathrm{I}_{u}$ from S1000s at locations of BL1 and BL2, respectively. All turbulence metrics in this figure are shown in logarithmic scale
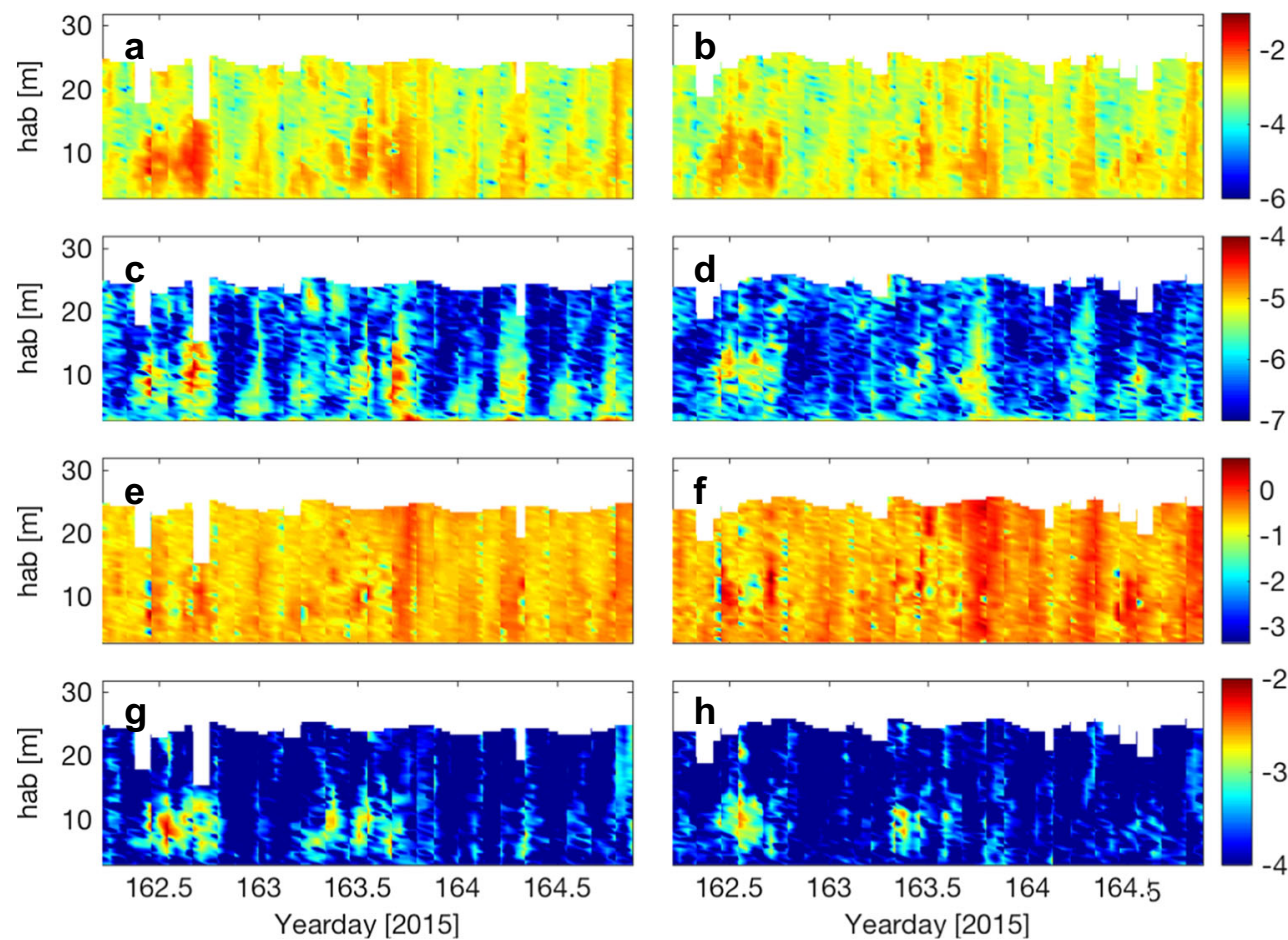

within the uppermost sampling volumes may be able to affect the ambient turbulent variances. The quasi-periodic temporal variability of $I_{u}$ at deeper levels can be attributed to the contribution from tides (with dominant period of $\mathrm{M}_{2}$ ).

\section{Conclusions}

We reported novel observational systems to measure ocean currents and high-resolution turbulence parameters, such as Reynolds stress and turbulent kinetic energy (TKE) throughout the water column. The experiment was conducted in close vicinity of FINO1 platform in the North Sea and near the Alpha Ventus wind farm, allowing us to study possible effects of farm on ocean currents and turbulence. Measurements performed from two bottom-mounted 5-beam Nortek Signature1000s (S1000s) deployed at two sites, one upwind of FINO1 and the other downwind of the wind farm. We presented the variance-method framework to estimate the turbulent stresses from S1000s in the presence of surface gravity wave orbital velocities and wave-induced instrumental tilt (i.e measurements from non-orthogonal axes). In order to account for the wave effect, we computed the covariances by integrating only the below-wave band of the measured turbulent cospectra. The below-wave motions approximately explained the majority of covariances for interior ocean sampling volumes. While applying this procedure underestimates the true RSs, comparisons of the surface/bottom friction velocities using uncorrected and corrected turbulence covariances to the estimates made by drag laws (Large and Pond 1981) indicated that the correction method substantially reduces (by approximately one order of magnitude) the wave-induced biases from turbulence measurements. In contrast to the mean flow which shows weak effects from the farm wake, profiles of Reynolds stresses (and non-dimensional drag coefficients) show relatively strong response to the surface forcing, gravity waves, tidal forcing and the wind farm wake-induced distortions in both boundary layers near the surface and bottom. We observed similar, but less pronounced, trends for vertical distributions of other turbulence parameters (e.g TKE production, turbulent intensity and anisotropy ratio). Although comparisons were limited to only 3 days because of a transducer failure on one of the S1000s, this investigation provides invaluable information about the wave-current and the wave-turbulence interactions in the vicinity of offshore structures which are crucial in the design and optimisation of marine energy devices and in turbulence modelling and analysis.

Acknowledgments The OBLEX-F1 field campaign has been performed under the Norwegian Centre for Offshore Wind Energy (NORCOWE) funded by the Research Council of Norway (RCN 193821), the Offshore Boundary Layer Observatory (OBLO) project (project no. RCN 227777) and the Norwegian e-infrastructure NorStore (project no. RCN: NS9060K). OBLEX-F1 was coordinated in collaboration between the University of Bergen (Geophysical Institute) and Christian Michelsen Research AS (project executing organisation). The Federal Maritime and Hydrographic Agency of Germany (BSH) is acknowledged for providing the FINO1 reference data through the FINO database at http://fino.bshde/. The FINO project (research platforms in the North Sea and Baltic Sea) is funded by the BMU, the 
German Federal Ministry for the Environment, Nature Conservation, Building and Nuclear Safety in collaboration with Project Management Jülich GmbH (project no. 0325321). The FINO1 meteorological reference data were provided by Deutsches Windenergi Institut (DEWI) and the FINO1 oceanographic reference data were provided by the BSH. We also thank DEWI for providing the FINO1 highresolution sonic anemometer data and the FINO1 platform operator Forschungs- und Entwicklungszentrum Fachhochschule Kiel GmbH (FuE Kiel GmbH) for their support (project no. 0329905E). We thank Steffen Howorek and Andreas Gudi (FuE Kiel GmbH) and Benny Svardal, Stian Stavland and Martin Flügge (all CMR) for their invaluable support in deploying and maintaining the meteorological instrumentation during the campaign. We also thank the crew of RV Håkon Mosby, Helge T. Bryhni and Steinar Myking for their professional deployment and retrieval of the oceanographic instrumentations. I would like also to express my gratitude to Jim Thomson for sharing his recent works on S1000 (Guerra and Thomson 2017) before starting the publishing process of this paper.

Open Access This article is distributed under the terms of the Creative Commons Attribution 4.0 International License (http:// creativecommons.org/licenses/by/4.0/), which permits unrestricted use, distribution, and reproduction in any medium, provided you give appropriate credit to the original author(s) and the source, provide a link to the Creative Commons license, and indicate if changes were made.

\section{References}

Bakhoday-Paskyabi M, Bryhni HT, Reuder J, Fer I (2015) Lagrangian measurement of waves and near surface turbulence from acoustic instruments. Energ Proc 80:141-150

Bakhoday-Paskyabi M, Fer I (2014) Turbulence structure in the upper ocean: a comparative study of observations and modelling. Ocean Dyn 64:611-631

Dewey RK, Stringer S (2007) Reynolds stresses and turbulent kinetic energy estimates from various ADCP beam configurations: Theory. J Phys Oceanogr 2:1-35

Feddersen F, Williams A (2007) Direct estimation of the Reynolds stress vertical structure in the nearshore. J Atmos Ocean Tech 24:102-116

Fer I, Bakhoday-Paskyabi M (2014) Autonomous ocean turbulence measurements using shear probes on a moored instrument. J Atmos Ocean Tech 31(2):474-490

Guerra M, Thomson J (2017) Turbulence measurements from fivebeam acoustic doppler current profilers. J Atmos Ocean Tech 34:1267-1284. https://doi.org/10.1175/JTECH-D-16-0148.1

Gargett A (1994) Observing turbulence with a modified acoustic Doppler current profiler. J Atmos Ocean Tech 11:1592-1610

Gerbi G, Trowbridge J, Edson J, Plueddemann A, Terray E, Fredericks $\mathrm{J}$ (2008) Measurements of momentum and heat transfer across the air-sea interface. J Phys Oceanogr 38:1054-1072

Howarth MJ, Souza AJ (2005) Reynolds stress observations in continental shelf seas. Deep-Sea Res 52:1075-1086
Kirincich A, Lentz S, Gerbi G (2010) Calculating Reynolds stresses from ADCP measurements in the presence of surface gravity waves using the cospectra fit method. J Atmos Ocean Tech 27:889-907

Large WG, Pond S (1981) Open ocean momentum flux measurements in moderate to strong winds. J Phys Oceanogr 11:324-336

Leibovich S (1983) The form and dynamics of Langmuir circulations. Annu Rev Fluid Mech 15:391-427

Lohrmann A, Hackett B, Roed LD (1990) High resolution measurements of turbulence, velocity, and stress using a pulse-to-pulse coherent sonar. J Atmos Ocean Tech 7:19-37

Lu Y, Lueck RG (1999) Using a broadband ADCP in a tidal channel. part II: Turbulence. J Atmos Ocean Tech 16:1568-1579

Lumley JL, Terray EA (1983) Kinematics of turbulence convected by a random wave field. J Phys Oceanogr 13:2000-2007

Nielsen P (1992) Costal bottom boundary layers and sediment transport. World Scientifc, New Jersey, p 324

Nystrom EA, Rehmann CR, Oberg M, Oberg KA (2007) Evaluation of mean velocity and turbulence measurements with ADCPs. J Hydraul Eng 133:1310-1318

Osalusi E, Side J, Harris R (2009) Reynolds stress and turbulence estimates in bottom boundary layer of fall of Warness. Intern Comm Heat Mass Transf 36:412-421

Peters H, Lee CM, Orlic' M, Dorman CE (2007) Turbulence in the wintertime Northern Adriatic Sea under strong atmospheric forcing. J Geophys Res 112:21

Rosman J, Gerbi G (2017) Interpreting fixed-location observations of turbulence advected by waves: insights from spectral models. Phys Oceanogr 47:909-931. https://doi.org/10.1175/JPO-D-150249.1

Rosman J, Hench J, Koseff J, Monismith S (2008) Extracting Reynolds stresses from acoustic Doppler current profiler measurements in wave-dominated environments. J Atmos Ocean Tech 25:286-306

Shaw W, Trowbridge J (2001) The direct estimation of near-bottom turbulent fluxes in the presence of energetic wave motions. J Atmos Ocean Tech 18:1540-1557

Teixeira MAC, Belcher S (2002) On the distortion of turbulence by a progressive surface wave. J Fluid Mech 458:229-267

Terray EA, Donelan MAYC, Agrawal WM, Kahma KK, Williams AJ, Hwang PA, Kitaigorodski SA (1996) Drennan Estimates of kinetic energy dissipation under breaking waves. J Phys Oceanogr 26:792-807

Thomson J (2012) Wave breaking dissipation observed with "SWIFT" drifters. J Atmos Ocean Tech 29:1866-1883

Thomson J, Polagye B, Durgesh V, Richmond M (2012) Measurements of turbulence at two tidal energy sites in Puget Sound. J Ocean Eng 37(3):363-374

Trowbridge J (1998) On a technique for measurement of turbulent shear stress in the presence of surface waves. J Atmos Ocean Tech 15:290-298

Whipple AC, Luettich RA, Seim HE (2006) Measurements of Reynolds stress in a wind-driven lagoonal estuary. Ocean Dyn 21:169-185

Wiles PJ, Rippeth TP, Simpson JH, Hendricks PJ (2006) A novel technique for measuring the rate of turbulent dissipation in the marine environment. Geophys Res Lett 33:15 